\title{
Stashing the stops in multijet events at the LHC
}

\author{
Sara Diglio, ${ }^{1, a}$ Lorenzo Feligioni, ${ }^{1, b}$ and Gilbert Moultaka ${ }^{2, c}$ \\ ${ }^{1}$ Centre de Physique des Particules de Marseille (CPPM), \\ UMR 7346 IN2P3-Univ. Aix-Marseille, Marseille, F-France \\ ${ }^{2}$ Laboratoire Charles Coulomb (L2C), \\ UMR 5221 CNRS-Université de Montpellier, Montpellier, F-France
}

(Dated: July 27, 2021) 


\begin{abstract}
While the presence of a light stop is increasingly disfavored by the experimental limits set on R-parity conserving scenarios, the naturalness of supersymmetry could still be safely concealed in the more challenging final states predicted by the existence of non-null R-parity violating couplings. Although R-parity violating signatures are extensively looked for at the Large Hadron Collider, these searches always assume $100 \%$ branching ratios for the direct decays of supersymmetric particles into Standard Model ones. In this paper we scrutinize the implications of relaxing this assumption by focusing on one motivated scenario where the lightest stop is heavier than a chargino and a neutralino. Considering a class of R-parity baryon number violating couplings, we show on general grounds that while the direct decay of the stop into Standard Model particles is dominant for large values of these couplings, smaller values give rise, instead, to the dominance of a plethora of longer decay chains and richer final states that have been so far barely analyzed at the LHC, thus weakening the impact of the present experimental stop mass limits. We characterize the case for R-parity baryon number violating couplings in the $10^{-7}-10^{-1}$ range, in two different benchmark points scenarios within the model-independent setting of the low-energy phenomenological Minimal Supersymmetric Standard Model. We identify the different relevant experimental signatures from stop pair production and decays, estimate the corresponding proton-proton cross sections at $\sqrt{s}=14 \mathrm{TeV}$ and discuss signal versus background issues.
\end{abstract}

PACS numbers: 12.60.-i;12.60.Jv;14.80.-j;14.80.Ly

Keywords: supersymmetry; R-parity violation; top squarks; LHC

\footnotetext{
${ }^{a}$ Current address: SUBATECH, Ecole des Mines de Nantes, CNRS/IN2P3, Université de Nantes, Nantes, France; electronic address: diglio@subatech.in2p3.fr

${ }^{b}$ Electronic address: lorenzo@cppm.in2p3.fr

cElectronic address: gilbert.moultaka@umontpellier.fr; corresponding author
} 


\section{INTRODUCTION}

The discovery at the Large Hadron Collider (LHC) of a weakly-coupled, spin-0 particle compatible with the Higgs boson [1-4], by both ATLAS [5] and CMS [6] collaborations, with a mass of approximately $125 \mathrm{GeV}$ [7], constrains all theoretical extensions to the Standard Model (SM) that aim at a mechanism for spontaneous Electroweak Symmetry Breaking (EWSB) relieved of the naturalness problem. In the coming years the measurements of the properties of this new particle will shed further light on the possibility of new physics at the $\mathrm{TeV}$ scale. While the presence of a new class of phenomena at the $\mathrm{TeV}$ scale is predicted by a large variety of models which address the various theoretical shortcomings of the SM, the LHC Run 1 and first Run 2 data sets analysed so far gave no evidence for new physics Beyond the Standard Model (BSM). Indirect manifestations might be hiding in heavy flavor rare decays anomalies reported by $\mathrm{LHCb}[8,9]$ with moderate to sizable statistical significance [10], and the well established neutrino oscillation phenomena [11] can be viewed as clear indications for the need for BSM physics [12].

Supersymmetry (SUSY) [13-18] has long been considered to be an elegant way of triggering the EWSB, relating it radiatively through perturbative quantum effects to possible new physics at much higher scales, such as Grand Unification, while stabilizing the various scales without unnatural fine-tunings. It can also provide in its R-parity conserving (RPC) version several dark matter candidates, the most popular being a neutralino when the lightest supersymmetric particle (LSP). Nonetheless, the naturalness of the Higgs potential favors light third-generation squarks whose RPC striking signatures have yet to be observed at hadron colliders, pushing the limits on the mass of such particles at the boundary of what is accepted to be natural. This could be a hint that the role of SUSY as a panacea for all SM standing problems should be revised. In particular if R-parity violating (RPV) operators $[19,20]$ in the superpotential are not artificially suppressed to allow for instance for a neutralino dark matter, RPV SUSY could be welcome for a natural EWSB since most of LHC constraints based on searches for missing energy signatures would not be valid anymore. One thus expects the interest in RPV SUSY searches at the LHC to build up significantly in the coming years [21].

From the theoretical point of view, it is attractive to view R-parity breaking as a dynamical issue. The magnitude of the RPV couplings could then be related to residual low-energy 
effects of some ultraviolet completions of the minimal SUSY extension of the Standard Model, see e.g. [22, 23] for recent reviews. On a more fundamental level, whether R-parity is conserved or not as a residual discrete symmetry of continuous R-symmetries, could also depend on the breaking mechanisms of the latter, which is an open question intimately related to the origin of SUSY breaking itself [24]. The presence of RPV operators with small couplings, but still sufficiently large to trigger prompt decays within the detector, is thus not unlikely. It can also preserve some of the appealing features of the RPC scenarios; e.g. a very light metastable gravitino can provide a viable dark matter candidate, and the stability of the proton can be protected by other discrete symmetries [25].

Limits on RPV scenarios have been given by ATLAS [26-32] and CMS [33-41]. These limits rely on simplifying model assumptions. In particular, the mass limits on the lighter stop assume in the case of hadronic stop RPV decays $100 \%$ branching ratio into two body final states $[31,32,41]$. It follows that, apart from the qualitative requirement of prompt decays, the derived limits are independent of the size of the RPV couplings themselves, and thus insensitive to the experimental limits on the latter [42]. While this assumption is clearly valid if the stop were the LSP, it calls for more model-dependence in the opposite case.

It has been pointed out in Ref. [43] that busier final states with high b-quark multiplicities, not looked for by the LHC experiments so far, can become the dominant stop decay channels in regions of the parameter space where part of the neutralino/chargino sector is lighter than the lightest stop, thus mitigating the present LHC limits. Furthermore, as shown in Refs. [44-46], existing experimental searches performed at the LHC, such as di-jet resonant production, top-quark pairs, four-tops and displaced decays, can be re-interpreted as limits for a class of RPV couplings involving the stop and SM quarks.

In the present paper we go a step further by considering extensively the sensitivity to the magnitudes of the RPV couplings for stop-pairs production. This pinpoints the critical role of the size of these couplings in unveiling the final states that are dominant among all the different combinations of stop decay chains. It also unfolds the experimental strategy to be sensitive to stop pair production and decays, spanning several orders of magnitude for the value of the RPV couplings.

The rest of the paper is organized as follows: in Section II we recall the main theoretical ingredients of the RPV sector of the Minimal Supersymmetric Standard Model (RPVMSSM), as well as the present LHC limits on RPV stop searches, discussing possible new 
search channels, and give the simplifying model assumptions we make. In Section III we describe the general features of the stop pair production and decays, classify all the possible decay channels triggered by R-parity violation and motivate the allowed range of the corresponding couplings. In Section IV we give an analytical discussion of the sensitivity to the considered RPV coupling, while in Section V we identify two classes of benchmark points of the model. The stop pair production total cross-section and decay channels for these two benchmark points are evaluated in Section VI illustrating quantitatively the phenomenological sensitivities to the RPV coupling and to the stop-chargino mass splitting. Section VII is devoted to a discussion of the signal and background issues for each of the promising final states. Finally, the conclusion and prospectives are given in Section VIII.

\section{MODEL ASSUMPTIONS}

\section{A. RPV-MSSM}

The superpotential of the RPV-MSSM (see for instance [42]) has three distinct parts:

$$
W_{\mathrm{RPV}}=W_{\mathrm{RPC}}+W_{\psi}+W_{\not B}
$$

The R-parity conserving part,

$$
W_{\mathrm{RPC}}=\left(Y^{L}\right)_{i j} \hat{L}_{i} \cdot \hat{H}_{1} \hat{E}_{j}^{c}+\left(Y^{D}\right)_{i j} \hat{Q}_{i} \cdot \hat{H}_{1} \hat{D}_{j}^{c}+\left(Y^{U}\right)_{i j} \hat{Q}_{i} \cdot \hat{H}_{2} \hat{U}_{j}^{c}+\mu \hat{H}_{2} \cdot \hat{H}_{1}
$$

involves the Yukawa coupling matrices $Y^{L}, Y^{D}, Y^{U}$ and the Higgs mixing parameter $\mu$. The R-parity violating part, $W_{\not}+W_{B}$, splits into a lepton number violating sector involving bilinear and trilinear couplings,

$$
W_{\psi}=\frac{1}{2} \lambda_{i j k} \hat{L}_{i} \cdot \hat{L}_{j} \hat{E}_{k}^{c}+\lambda_{i j k}^{\prime} \hat{L}_{i} \cdot \hat{Q}_{j} \hat{D}_{k}^{c}+\mu_{i} \hat{L}_{i} \cdot \hat{H}_{2}
$$

and a baryon number violating sector involving trilinear couplings,

$$
W_{\not B}=\frac{1}{2} \lambda_{i j k}^{\prime \prime} \hat{U}_{i}^{\alpha c} \hat{D}_{j}^{\beta c} \hat{D}_{k}^{\gamma c} \epsilon_{\alpha \beta \gamma} .
$$

The chiral superfields $\hat{L}$ and $\hat{Q}$ denote respectively the lepton and quark $S U(2)$ doublets, $\hat{E}, \hat{D}$ and $\hat{U}$ the corresponding singlets, and $\hat{H}_{1}$ and $\hat{H}_{2}$ are the two Higgs doublets, together with their conventional $U(1)_{Y}$ hypercharges. Summation over repeated indices is understood 
in all the above expressions where $\alpha, \beta, \gamma=1,2,3$ denote the $S U(3)$ color indices, the dots $\left(A \cdot B \equiv \epsilon_{a b} A^{a} B^{b}\right)$ define $\mathrm{SU}(2)$ invariants, the $i, j, k=1,2,3$ are generation indices, and $c$ indicates charge conjugation. Also the trilinear RPV couplings should satisfy the relation:

$$
\lambda_{i j k}=-\lambda_{j i k} \text { and } \lambda_{i j k}^{\prime \prime}=-\lambda_{i k j}^{\prime \prime}
$$

as an immediate consequence of the antisymmetry of the $\epsilon_{a b}$ and $\epsilon_{\alpha \beta \gamma}$ symbols respectively.

Recall that to account for SUSY breaking, assumed to be soft in the visible sector, the low energy MSSM is expected to have additional RPC and RPV terms in the Lagrangian density with the following general structure,

$$
\mathcal{L}_{\mathrm{RPC}}^{\mathrm{soft}}=-V_{\mathrm{RPC}}^{\mathrm{soft}}-\frac{1}{2}\left(M_{1} \tilde{B} \tilde{B}+M_{2} \tilde{W} \tilde{W}+M_{3} \tilde{g} \tilde{g}\right)
$$

where

$$
\begin{aligned}
V_{\mathrm{RPC}}^{\mathrm{soft}}= & \left(m_{\tilde{Q}}^{2}\right)_{i j} \tilde{Q}_{i}^{\dagger} \tilde{Q}_{j}+\left(m_{\tilde{U}}^{2}\right)_{i j} \tilde{U}_{i}^{\dagger} \tilde{U}_{j}+\left(m_{\tilde{D}}^{2}\right)_{i j} \tilde{D}_{i}^{\dagger} \tilde{D}_{j}+\left(m_{\tilde{L}}^{2}\right)_{i j} \tilde{L}_{i}^{\dagger} \tilde{L}_{j}+\left(m_{\tilde{E}}^{2}\right)_{i j} \tilde{E}_{i}^{\dagger} \tilde{E}_{j}+m_{H_{1}}^{2}\left|\tilde{H}_{1}\right|^{2} \\
& +m_{H_{2}}^{2}\left|\tilde{H}_{2}\right|^{2}+\left(\left(T^{l}\right)_{i j} \tilde{L}_{i} \cdot \tilde{H}_{1} \tilde{E}_{j}^{c}+\left(T^{d}\right)_{i j} \tilde{Q}_{i} \cdot \tilde{H}_{1} \tilde{D}_{j}^{c}+\left(T^{u}\right)_{i j} \tilde{Q}_{i} \cdot \tilde{H}_{2} \tilde{U}_{j}^{c}+B_{\mu} \tilde{H}_{2} \cdot \tilde{H}_{1}+\text { h.c. }\right)
\end{aligned}
$$

involves the RPC soft SUSY breaking scalar masses, trilinear couplings and Higgs mixing, and

$$
\mathcal{L}_{\mathrm{RPV}}^{\mathrm{soft}}=-V_{\not}^{\mathrm{soft}}-V_{\not B}^{\mathrm{soft}}
$$

where

$$
V_{\not}^{\text {soft }}=\frac{1}{2} T_{i j k} \tilde{L}_{i} \cdot \tilde{L}_{j} \tilde{E}_{k}^{c}+T_{i j k}^{\prime} \tilde{L}_{i} \cdot \tilde{Q}_{j} \tilde{D}_{k}^{c}+B_{i} \tilde{L}_{i} \cdot \tilde{H}_{2}+\tilde{m}_{1 i}^{2} \tilde{H}_{1}^{\dagger} \tilde{L}_{i}+\text { h.c. },
$$

and

$$
V_{\not B}^{\text {soft }}=\frac{1}{2} T_{i j k}^{\prime \prime} \tilde{U}_{i}^{\alpha c} \tilde{D}_{j}^{\beta c} \tilde{D}_{k}^{\gamma c} \epsilon_{\alpha \beta \gamma}+\text { h.c. }
$$

involve respectively the lepton and baryon number violating soft SUSY breaking bilinear and trilinear couplings. In Eq. (2.6) the twiddled fields denote the $U(1)_{Y}, S U(2)_{L}$, and $S U(3)$ gauginos in the Weyl representation where we have suppressed the gauge indices, and $M_{1}, M_{2}, M_{3}$ denote their soft masses. The fields in Eqs. $(2.7,2.9,2.10)$ are the scalar components of the chiral superfields entering the superpotentials $(2.2-2.4)$ and the $\mathrm{m}^{2}$ 's, $B_{\mu}, B_{i}, T^{l, d, u}, T, T^{\prime} T^{\prime \prime}$ are the bilinear and trilinear soft-susy breaking parameters. We define also $\tan \beta \equiv \frac{v_{2}}{v_{1}}$, the ratio of the vacuum expectation values developed by $H_{2}$ and $H_{1}$ after EWSB. 
In the sequel we do not rely on specific high scale model assumptions which can trigger the EWSB and correlate the various low-energy SUSY preserving and soft breaking parameters, or possibly provide a dynamical origin to the RPV couplings [22, 23]. Given the lowenergy phenomenological assumptions we rely on, the process of stop production and decays under consideration depends only on a reduced set of MSSM parameters insensitive to such correlations. Furthermore, we assume conservatively minimal flavor violation (MFV) [47], since the heavy versus light quark content of the final states is instrumental to our study.

\section{B. LHC searches and new channels}

The likeliness of a relatively light stop, motivated by natural SUSY and a large mass splitting between the two stop states that could account for the observed Higgs boson mass (at least within the MSSM), together with the more general expectation that the third (s)quark generation plays a central role in triggering the electroweak symmetry breaking, makes the search for light stops particularly compelling. This is true both in RPC and RPV scenarios. The present LHC mass limits from direct production in the RPC scenarios are of order $800 \mathrm{GeV}$ [48-51] and the exploitable range is expected to cross the $\mathrm{TeV}$ scale towards the end of Run 2. Moreover, some of the all-leptonic RPV searches have already increased this limit in some cases up to $1020 \mathrm{GeV}$ [33]. Lighter stops could however still be hiding in the all-hadronic channels final states with very low missing energy, as would be typically the case in RPV scenarios if dominated by baryon number violating couplings $\lambda_{33 i}^{\prime \prime}$, cf. Eq. (2.4). Searches for directly produced stop pairs each decaying into one jet originating from a $b$ and one jet from a light quark with the data collected in 2012 at $\sqrt{s}=8 \mathrm{TeV}$ and in 2015 at $\sqrt{s}=13 \mathrm{TeV}$ lead to exclusion mass limits in the range 100-380 GeV by the CMS [41] and ATLAS [31, 32] collaborations.

Both ATLAS [26-30] and CMS [33-40] have also looked for signatures of RPV scenarios through either gluino decays assuming baryon number violating couplings, or squark decays assuming lepton number violating bilinear and trilinear couplings. The ensuing mass limits for the gluino and first and second generation squarks range from $800 \mathrm{GeV}$ up to 1.9 $\mathrm{TeV}$ depending on the model assumptions.

It is important to keep in mind that the limits quoted above assume the RPV decays to proceed through the shortest decay chains. In particular the ones on direct production of 
stops decaying through baryon number violating couplings, are derived under the assumption of $100 \%$ decay into a bottom and a light quark. These limits carry thus some modeldependence irrespective of whether lepton number violating decays are ignored or not. As observed in Ref. [43], if the stop is not the LSP in parts of the parameter space motivated by natural SUSY, then its decays may become dominated by channels with higher $b$-quark multiplicities. In this case, a different experimental strategy is called for when looking for a signal or setting limits, thus putting into more perspective the meaning and reach of the present experimental limits on light stops. However, it is to be stressed that even in Ref. [43] a 100\% decay in the final states under consideration is assumed, this time not for the decaying mother stop itself but for the subsequent decay of the intermediate on-shell chargino present in the decay chain. As noted in the introduction, such an assumption makes the processes and the experimental limits insensitive to the magnitude of the relevant RPV couplings. Not only is it desirable to be able to set limits on these couplings as well, but in fact, in the configurations where the LSP is neither a squark nor a slepton, the branching fractions of the various RPV decays of the latter depend necessarily on the magnitudes of the RPV couplings. That this is to be expected on general grounds can be seen from the simple fact that in the limit of vanishing RPV couplings the RPC theory should be recovered smoothly. Indeed, in this limit, of all the RPV signal processes only the ones that tend to the RPC signals, i.e. containing an on-shell long-lived LSP in the decay chain, will survive. This implies that when decreasing the RPV couplings a crossover in favor of the decays containing the LSP must occur at some point. Moreover, in the regions where they become sizable, the latter channels tend to be less sensitive to the RPV couplings since the LSP decays only through RPV channels, thus with branching ratio 1 to the relevant final states. The only limitation is that the RPV couplings should remain sufficiently large for the LSP to decay within the detector, otherwise the RPC search limits become effective.

Put differently, assuming a branching ratio of 1 for a given decay channel implicitly entails a given range of the RPV couplings, that would further depend on the mass spectrum and RPC couplings of the particles involved in the decay. This observation has two consequences:

- while all the quoted present experimental limits on RPV scenarios have obviously some model-dependence, the sensitivity to the RPV couplings exacerbates this modeldependence; 
- higher jet and/or lepton multiplicity decays probe smaller (even tiny) RPV couplings benefiting in the same time from a reduced SM background.

The aim of the subsequent sections is to demonstrate the above general features quantitatively in the case of baryon number violating RPV couplings $\lambda_{331}^{\prime \prime}$ or $\lambda_{332}^{\prime \prime}$ that trigger the decay of stops leading to $b$-quarks, light quarks and possibly leptons in the final states.

\section{C. mass spectrum}

In this section we describe the simplified working assumptions made in the paper:

(i) $\lambda_{33 i}^{\prime \prime}$, with $i=1$ or 2 , is the only non-vanishing RPV coupling,

(ii) the light part of the SUSY spectrum is composed of one stop, one chargino, two neutralinos and the lightest CP-even Higgs (referred to respectively as $\tilde{t}, \chi^{+}, \chi^{0} / \chi_{2}^{0}$ the lighter/heavier neutralino and $h^{0}$ the SM-like Higgs throughout the paper). All other SUSY and Higgs particles, except possibly for the gluino, are assumed to be too heavy to be produced at the LHC,

(iii) the RPV-MSSM-LSP is the lightest neutralino $\chi^{0}$.

A few comments are in order here. Assumptions (i), (ii), (iii) are not mandatory for the validity of the general message we convey in this paper regarding the final-state-dependent sensitivity to the RPV couplings. They serve as a concrete illustration in one possible physically interesting configuration. Assumption (i) can be seen as an idealization of some generic assumptions such as MFV where baryon number violating RPV couplings containing 1st and 2nd generation indices are suppressed with respect to $\lambda_{332}^{\prime \prime}$ (or $\left.\lambda_{331}^{\prime \prime}\right)[52,53]$. Alternatively, it could result from a dynamical collective effect due to the running of several RPV couplings from a common value at some very high scale down to the electroweak scale where $\lambda_{332}^{\prime \prime}$ becomes much larger than the other couplings [54, 55]. In fact, our analysis does not depend crucially on the single RPV coupling dominance assumption: indeed, combined with assumption (ii), assumption (i) is not particularly restrictive given the hadronic final states and parameter space under consideration. For one thing, $\lambda_{33 i}^{\prime \prime}$ can be viewed as ac-

counting for the combination $\sqrt{\left(\lambda_{332}^{\prime \prime}\right)^{2}+\left(\lambda_{331}^{\prime \prime}\right)^{2}}$ since at present hadron colliders light $d$ - 
and $s$-quark productions are indistinguishable. ${ }^{1}$ For another, most of the lepton number violating couplings in Eq. (2.3) do not contribute to the final states under consideration, or else are irrelevant due to the assumed heaviness of the squarks and sleptons. The only possible exception is the set of $\lambda_{i j 3}^{\prime}$ couplings that induce $\tilde{t}$ decays into bottom quark and a lepton. This channel would however be suppressed for a small left-handed component of the lightest stop, and in any case can be vetoed as it leads to final states with leptons and no light quarks, different from the ones we study. Finally the baryon number violating couplings $\lambda_{132}^{\prime \prime}, \lambda_{232}^{\prime \prime}$ can in principle contribute to final states containing $b$ - and light quarks through the flavor mixing of the 3rd generation with the 1st and 2nd generation squarks (current states). However this mixing is very small for the SUSY spectrum we consider which suppresses the sensitivity to these couplings altogether. Thus most of the RPV couplings could still be non-vanishing without affecting our analysis. Assumption (ii) can be motivated on one hand by simplicity, with only a small part of the MSSM spectrum to deal with phenomenologically, and on the other by the need to account for the light CP-even Higgs mass while keeping at a relatively moderate level the fine-tuning required to get the electroweak scale from the radiative electroweak symmetry breaking, see e.g. [57]. It should be stressed however that the latter naturalness criterion being more a practical guide than a physics principle, the actual realisation of the low lying states of supersymmetry could well be through quite different configurations than the ones motivated by naturalness.

As concerns assumption (iii), obviously not motivated by dark matter issues since the RPV-MSSM-LSP is unstable and assumed to decay promptly, its aim is to remain as close as possible to the conventional spectrum configurationns for which most of the present experimental bounds for RPC scenarios have been established. In particular this allows to relate in a well defined way to the latter bounds whenever $\lambda_{33 i}^{\prime \prime}$ becomes too small for the $\chi^{0}$ to decay within the detector. Still it is important from a more general perspective to assess the dark matter candidates in the RPV context. We only note here that among the possible scenarios a light gravitino, being for that matter the true LSP (leaving the $\chi^{0}$ as the RPV-MSSM-LSP), can indeed provide a good metastable candidate even for moderately large RPV couplings of order $10^{-2}$ or larger, for sufficiently heavy sfermions [58-60]. In fact,

\footnotetext{
${ }^{1}$ This correspondence is valid up to indirect effects originating from RPV induced loop corrections to the $\tilde{t}$ mass [56]. These effects remain, however, negligibly small in the $\lambda_{33 i}^{\prime \prime}$ range we consider.
} 
with assumption (i), a gravitino lighter than twice the $b$-quark mass would be even totally stable.

Besides assumptions (i), (ii),(iii), we focus mainly, though not exclusively, on the MSSM parameter regions that are consistent with the following mass configuration:

$$
\begin{aligned}
& m_{\tilde{t}} \gtrsim m_{\chi_{2}^{0}} \gtrsim m_{\chi^{+}} \gtrsim m_{\chi^{0}}>m_{t}, \\
& m_{\tilde{t}}-m_{\chi^{0}}<m_{t}, \\
& m_{\tilde{t}}-m_{\chi^{+}}>m_{b} .
\end{aligned}
$$

Such a configuration has been already considered in Ref. [43] to illustrate the relevance of multi $b$-quark final states when an on-shell chargino is present in the stop decay chain. In the present work we stress the relevance of the longer decay chain not considered previously, containing on-shell chargino and neutralino, and in particular the importance of the magnitude of $\lambda_{33 i}^{\prime \prime}$ in selecting the stop decay channels that actually dominate. Note also the presence of two neutralinos in the low energy spectrum. This is unavoidable when the chargino/neutralino light sector is assumed to be Higgsino-like as we do: in the limit $M_{1} \simeq M_{2} \gg \mu \gg m_{W}$ and $\tan \beta \gg 1$ one finds $m_{\chi_{2}^{0}}-m_{\chi^{+}} \sim m_{\chi^{+}}-m_{\chi^{0}} \simeq \frac{5}{8} \frac{m_{W}^{2}}{M_{1}}$ up to loop corrections, which corresponds to a compressed spectrum satisfying the mass hierarchy in the chargino/neutralino sector as given in Eq. (2.11). However, as long as the configuration in Eq. (2.12) is satisfied the second neutralino, $\chi_{2}^{0}$, does not contribute significantly to the stop decay since it enters the decay chain only off shell, and is neglected throughout the study.

\section{STOP PRODUCTION AND DECAYS}

\section{A. pair production}

The stop pair production at the LHC, $p p \rightarrow \tilde{\tilde{t}}+X$, proceeds mainly through gluon-gluon fusion QCD processes, see [61-63] and references therein. While quark-anti-quark partonic contributions are subdominant at LHC energies, there could also be interesting single, or same-sign pair, stop (associated) productions respectively through RPV quark-quark processes or QCD gluon-gluon processes [64], [46]. Some of these channels are suppressed in 
our case, either because $\lambda_{3 k i}^{\prime \prime}$ with $k \neq 3$ are assumed to be vanishing or due to the assumed heaviness of the gluino and first and second squark generations. The single stop production and decays can already constrain parts of the parameter space for a light LSP as shown in [46]. Note however that the corresponding production cross-section becomes subdominent as compared to the pair production when $\lambda_{33 i}^{\prime \prime}$ is taken $\lesssim O\left(10^{-2}\right)$ and $m_{\tilde{t}} \gtrsim 500 \mathrm{GeV}$, and even totally suppressed for the much smaller values of $\lambda_{33 i}^{\prime \prime}$ that we consider in this paper.

\section{B. RPV final states}

Given the mass configurations described in Eqs. (2.11-2.13), the leading RPV and RPC $\tilde{t}$ decays are respectively $\tilde{t} \rightarrow \bar{b} \bar{d}_{i}$ and $\tilde{t} \rightarrow \chi^{+} b$, where $d_{i}$ with $i=1,2$, denotes respectively the $d$ - and $s$-quark. Other decay channels such as $\tilde{t} \rightarrow \chi^{0} t^{*} \rightarrow \chi^{0} b f_{1} \bar{f}_{1}^{\prime}$ or $\tilde{t} \rightarrow h^{0} \tilde{t}^{*} \rightarrow h^{0} \bar{b} \bar{d}_{i}$ (where $f_{1}$ and $\bar{f}_{1}^{\prime}$ indicate SM fermions and the star off-shell states), are suppressed by the off-shellness of the (s)top quark. Note also that a potential enhancement of the Higgs channel by large soft-susy breaking trilinear coupling is suppressed when the $\tilde{t}$ is essentially right-handed. The subsequent leading RPV induced $\chi^{+}$decays are $\chi^{+} \rightarrow \tilde{t}^{*} \bar{b} \rightarrow \bar{b} \bar{b} \bar{d}_{i}$ and the much longer chain $\chi^{+} \rightarrow \chi^{0} W^{+*} \rightarrow \tilde{t}^{*} \bar{t}\left(\tilde{\bar{t}}^{*} t\right) W^{+*} \rightarrow \bar{b} \bar{d}_{i} \bar{t}\left(b d_{i} t\right) f \bar{f}^{\prime}$ with the top decaying ultimately to $b f_{1} \bar{f}_{1}^{\prime}$ and where we assumed $\chi^{0}$ decays through the shortest possible chain. The latter decay, $\chi^{0} \rightarrow \tilde{t}^{*} \bar{t}\left(\tilde{\bar{t}}^{*} t\right) \rightarrow \bar{b} \bar{d}_{i} \bar{b}\left(b d_{i} b\right) f_{1} \bar{f}_{1}^{\prime}$, is indeed dominant as a consequence of assumption (ii) of Section IIC. The other equally short chain $\chi^{0} \rightarrow \tilde{b}^{*} b\left(\tilde{\bar{b}}^{*} \bar{b}\right) \rightarrow \bar{t}^{(*)} \bar{d}_{i} b\left(t^{(*)} d_{i} \bar{b}\right)$ is suppressed for sufficiently heavy $\tilde{b}$. The longer chains $\chi^{0} \rightarrow \chi^{+*} W^{-(*)}\left(\chi^{-*} W^{+(*)}\right) \rightarrow \tilde{t^{*} \bar{b}} W^{-(*)}\left(\tilde{\bar{t}^{*}} b W^{+(*)}\right) \rightarrow \bar{b} \bar{d}_{i} \bar{b}\left(b d_{i} b\right) f \bar{f}^{\prime}$ or $\chi^{0} \rightarrow \chi^{+*} W^{-(*)}\left(\chi^{-*} W^{+(*)}\right) \rightarrow \tilde{\bar{b}}^{*} t W^{-(*)}\left(\tilde{b}^{*} \bar{t} W^{+(*)}\right) \rightarrow t d_{i} t\left(\bar{t} \bar{d}_{i} \bar{t}\right) f \bar{f}^{\prime}$ are obviously even further suppressed.

We have thus at hand the three different decay channels depicted in Figs. 1 (a), (b) and (c). We refer to these respectively as $\tilde{\mathbf{t}}-\mathbf{R P V}, \boldsymbol{\chi}-\mathbf{R P V}$ and $\mathbf{R P C}-$ like, to stress the fact that channel (a) is the direct RPV stop decay, channel (b) the shortest RPV cascade containing an (on-shell) chargino, and channel (c), defined as having an on-shell $\chi^{0}$ intermediate state, corresponds to the only surviving channel in the RPC limit $\lambda_{33 i}^{\prime \prime} \rightarrow 0$. It is to be noted that the latter channel has not been considered in [43].

Note that because of the nearly mass degenerate chargino and neutralino in our scenario, off-shell $W$ bosons from the RPC-likestop decay chain are produced with a too small 


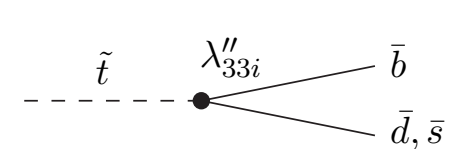

(a)

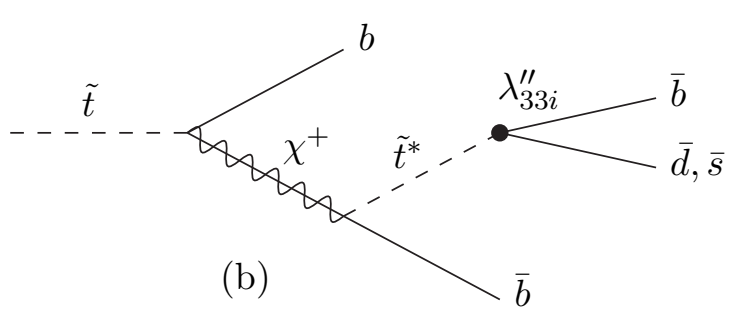

(b)

FIG. 1: Leading RPV stop decays assuming Eqs. (2.11-2.13); (a): direct RPV stop decay ( $\tilde{\mathbf{t}}-\mathbf{R P V}$ ), (b): shortest RPV cascade containing an (on-shell) chargino ( $\boldsymbol{\chi}$-RPV), (c): shortest RPV cascade containing an (on-shell) neutralino ( RPC-like); $f, f^{\prime}, f_{1}, f_{1}^{\prime}$ denote SM fermions and the oval encircles fermions too soft to be detected.

transverse momentum for their decay products to be reconstructed in Hight Energy Physics detectors. These are thus ignored in the following.

Since jets electric charges cannot be discriminated experimentally, we tag the various final states by their flavor content as follows:

- $\tilde{\mathbf{t}}-\mathbf{R P V} \equiv 1 b 1 j$,

- $\chi-\mathbf{R P V} \equiv 3 b 1 j$,

- RPC-like $\equiv 1 t 2 b 1 j$,

where $b(t)$ stands for the presence of a bottom-quark jet (top-quark) and $j$ indicates the presence of a light-quark jet. Since the RPC-like channels are characterized by the presence of a top quark in the decay chain followed by SM top decays, we have indicated only the presence of the top quark. We are thus left effectively with six different categories of final states corresponding to the decays of the produced stop and anti-stop as summarized in Table I. Final states with the same particle content (but opposite charges) are not duplicated in the table. We however continue to indicate explicitly the charges for definiteness when discussing the analytical structure of the cross-sections in Section IV. 


\begin{tabular}{|c|c|c|c|}
\hline$\overline{\tilde{\tau}}$ & $\tilde{\mathbf{t}}-\mathbf{R P V}$ & $\chi-\mathrm{RPV}$ & RPC-like \\
\hline$\tilde{t}-R P V$ & $2 b 2 j$ & $4 b 2 j$ & $1 t 3 b 2 j$ \\
\hline$\chi-\mathrm{RPV}$ & & $6 b 2 j$ & $1 t 5 b 2 j$ \\
\hline RPC-like & & & $2 t 4 b 2 j$ \\
\hline
\end{tabular}

TABLE I: The various final states corresponding to different contents of heavy $(b, t)$ quarks, and light $(d, s)$ quarks denoted generically by $j$, originating from the stop-anti-stop RPV decays; similar final states corresponding to interchanging the stop and anti-stop decays leading to the same particle content (irrespective of the electric charges) are listed only once.

\section{The $\lambda_{33 i}^{\prime \prime}$ range}

There exists a large set of upper bounds on the RPV couplings (see [42] for a detailed discussion), some of which involve $\lambda_{33 i}^{\prime \prime}$. Together with assumption (i) of Sec. II C, we allow in the sequel $\lambda_{33 i}^{\prime \prime}$ to vary in the range

$$
10^{-7} \lesssim\left|\lambda_{33 i}^{\prime \prime}\right| \lesssim 10^{-1}
$$

Experimental upper bounds on $\lambda_{331}^{\prime \prime}$ and $\lambda_{332}^{\prime \prime}$ are typically weaker than the ones involving only first and second generation, let alone the bounds on the lepton number violating couplings. Moreover, most of these bounds are on products of $\lambda_{33 i}^{\prime \prime}$ with other RPV couplings. Such bounds can thus be easily satisfied through assumption (i) of Sec. II C. There are also upper bounds set directly on $\lambda_{332}^{\prime \prime}$ and/or $\lambda_{331}^{\prime \prime}$, coming from constraints on the $Z$-boson hadronic width, neutron-anti-neutron oscillations and single nucleon decays: the first is $\mathcal{O}(1)$, the second and the third are model-dependent and are made easily compatible with the upper bound in Eq. (3.1) for squark masses $\gtrsim 500 \mathrm{GeV}$ (even more so for single nucleon decays assuming a gravitino mass $\gg 1 \mathrm{eV}$ or an axion scale $\gtrsim 10^{10} \mathrm{GeV}$ ). Likewise, the upper bound in Eq. (3.1) can be easily made compatible with bounds on the product $\left|\lambda_{331}^{\prime \prime}\left(\lambda_{332}{ }^{\prime \prime}\right)^{*}\right|$ obtained from $K^{0}-\bar{K}^{0}$ mixing for squark masses in the few hundred GeV range. All in all, the upper bound of Eq. (3.1) is only taken as a working assumption and could in principle 
be somewhat larger. Note however that values of $\lambda_{33 i}^{\prime \prime}$ much larger than $10^{-1}$ would lead to too large and negative loop corrections to the squared stop mass [56].

The lower bound in Eq. (3.1) is an estimate of the magnitude of $\lambda_{33 i}^{\prime \prime}$ that guarantees decays within the detector. Since in the configuration under study the lightest stop is not the lightest MSSM particle, one should consider not only the lifetime due to direct RPV two-body decay of the stop, Fig. 1(a), but also that of the daughter chargino due to its decay as given in Fig. 1(b), or the neutralino due to its decay as given in Fig. 1(c). In the absence of any prior about which channel among the $\tilde{\mathbf{t}}-\mathbf{R P V}, \boldsymbol{\chi}-\mathbf{R P V}$ or $\mathbf{R P C}$-like is dominant one should consider the most conservative bound, i.e. the longest decay length. The various $c \tau$ 's are (approximately) given by

$$
c \tau_{\tilde{t} \rightarrow b d_{i}}[\text { meter }] \simeq \frac{8.3 \times 10^{-18}}{\left|\lambda_{33 i}^{\prime \prime}\right|^{2}}\left(\frac{600 \mathrm{GeV}}{m_{\tilde{t}}}\right),
$$

for the direct RPV stop decay, where $d_{1,2}$ denote the first and second generation down quarks, and

$c \tau_{\chi^{0} \rightarrow t b d_{i}}[$ meter $] \simeq \frac{2.6 \times 10^{-16}}{\alpha_{\chi^{0}}\left|\lambda_{33 i}^{\prime \prime}\right|^{2}}\left(\frac{m_{\tilde{t}}}{600 \mathrm{GeV}}\right)^{4}\left(\frac{500 \mathrm{GeV}}{m_{\chi^{0}}}\right)^{5}\left(\left(1-r^{4}\right)\left(1-8 r^{2}+r^{4}\right)-24 r^{4} \log r\right)^{-1}$

for the Higgsino component of the neutralino RPV decay where we defined $\alpha_{\chi^{0}} \equiv \frac{g_{\chi^{0}}^{2}}{4 \pi}, g_{\chi^{0}}$ denoting the $\chi^{0}-\tilde{t}-t$ coupling, and $r \equiv \frac{m_{t}}{m_{\chi^{0}}}$ where $m_{t}$ is the top mass, and neglected $b$ - and light quark masses. ${ }^{2}$ In Eq. (3.3) we approximate the stop propagator by a point interaction which leads to an overestimate of the decay length and thus to a safe conservative bound, but we provide the exact integral over the three-body phase space taking into account the matrix element spinorial structure of the final state. The $c \tau$ corresponding to the chargino decay $\chi^{+} \rightarrow b b d_{i}$ is given by $2 \times c \tau_{\chi^{0} \rightarrow t b d_{i}}$ in the limit $m_{t} \rightarrow 0$ and with the proper substitution of

\footnotetext{
${ }^{2}$ In deriving these expressions we included consistently the color factors, averaged over the spin of the decaying particle and assumed the lightest stop to be essentially right-handed. (Note that some simple formulae for the neutralino decay length in the literature, e.g. Eq.(7.6) of Ref. [42], assume a pure photino content and do not apply in our case.) We also rely on the simplifying assumption of instantaneous decay at the mean lifetime, and travel of the decaying particle close to ( $70 \%$ of) the speed of light in the laboratory frame. A more accurate evaluation of the decay lengths should take into account boost factors from the actual mass and energy distributions of the decaying particles produced at various energies at the partonic level, as well as their lifetimes distribution.
} 
chargino mass and coupling, where the global factor two difference between the two $c \tau$ 's is due to the majorana nature of $\chi^{0}$. From Eqs. $(3.2,3.3)$ one has generically the hierarchy

$$
c \tau_{\tilde{t} \rightarrow b d_{i}} \ll c \tau_{\chi^{+} \rightarrow b b d_{i}} \lesssim c \tau_{\chi^{0} \rightarrow t b d_{i}},
$$

if $m_{\tilde{t}}>m_{\chi^{0}} \simeq m_{\chi^{+}} \lesssim 550 \mathrm{GeV}$ and $\alpha_{\chi^{0}}, \alpha_{\chi^{+}}<1$. The lower bound for $\left|\lambda_{33 i}^{\prime \prime}\right|$ is thus determined by the decay length of the neutralino provided that it corresponds to values of $\left|\lambda_{33 i}^{\prime \prime}\right|$ for which the stop decays containing a neutralino indeed dominate.

With a fiducial region of $c \tau \lesssim 3$ meters and taking $m_{\tilde{t}}=600 \mathrm{GeV}$, one has from Eq. (3.2) the lower bound $\left|\lambda_{33 i}^{\prime \prime}\right| \gtrsim 1.6 \times 10^{-9}$, while varying $m_{\chi^{0}} \simeq m_{\chi^{+}}$in the range $\left(600 \mathrm{GeV}-m_{t}\right)$ to $600 \mathrm{GeV}$, one obtains from Eq. (3.3) with a typical $\alpha_{\chi^{0}} \simeq 10^{-2}$ the stronger bound $\left|\lambda_{33 i}^{\prime \prime}\right| \gtrsim(0.8-2.4) \times 10^{-7}$. Of course, lighter stop and neutralino lead to more stringent lower bounds, e.g. $m_{\tilde{t}}=400 \mathrm{GeV}$ and $m_{\chi^{0}}=m_{\tilde{t}}-m_{t}$ would require $\left|\lambda_{33 i}^{\prime \prime}\right| \gtrsim 3.4 \times 10^{-6}$. However, a stop that light becomes barely compatible with our assumption that it is heavier than a chargino, since such a low mass configuration would start conflicting with limits on rare $B$-decays (see also the discussion in Section V 1).

When $m_{\chi^{0}} \simeq m_{\chi^{+}} \gtrsim 560 \mathrm{GeV}$ but still smaller than the stop mass, the 3-body phase space reduction in the $\chi^{0}$ decay width as compared to that in the $\chi^{+}$decay width, does not compensate anymore for the factor two difference between the two widths. As a result, the hierarchy of the chargino and neutralino $c \tau$ 's is reversed with respect to Eq. (3.4). However, the relevant lower bound for $\left|\lambda_{33 i}^{\prime \prime}\right|$ is still determined by the decay length of the neutralino. Indeed the chargino becomes detector-stable typically also for $\left|\lambda_{33 i}^{\prime \prime}\right|=\mathcal{O}\left(10^{-7}\right)$, where, as shown in the following Sections, the stop decay channels not containing a neutralino become highly suppressed.

Finally, note that we neglect altogether the gravitationally induced direct stop decay into a top-quark and a gravitino. This channel could lead to large missing energy in the final state. However, it is Planck scale suppressed unless the gravitino mass is in the deep sub-eV range [65]. As noted previously in this section, a gravitino much lighter than $1 \mathrm{eV}$ is disfavored by proton decay bounds, otherwise $\lambda_{331}^{\prime \prime}$ and $\lambda_{332}^{\prime \prime}$ would have to be typically much smaller than $\mathcal{O}\left(10^{-7}\right)$ where the LHC exclusion limits on RPC signatures apply. This suggests a rather heavy gravitino, for which stop decays with missing energy are not significant, and which is moreover welcome in scenarios of gravitino dark matter. One should however keep in mind that such stringent individual upper bounds on $\lambda_{331}^{\prime \prime}$ and $\lambda_{332}^{\prime \prime}$ from proton decay 
[66], can be relaxed through possible destructive interference if the two RPV couplings are allowed to be simultaneously non-vanishing, thus bringing them again within the lower part of the range given in Eq. (3.1). ${ }^{3}$

More generally, recasting experimental LHC limits on long-lived particle searches [67, 68] as done in $[45,46]$, constrains the various $c \tau^{\prime}$ 's to be in the millimeter range. Although the latter studies do not compare directly to ours, as they scan different mass spectra configurations, a $c \tau \simeq 3 \mathrm{~mm}$ for a decaying chargino LSP of $600 \mathrm{GeV}$ [45] would increase the lower bound in Eq. (3.1) to $\simeq 2.5 \times 10^{-6}$.

\section{NARROW WIDTH APPROXIMATION}

A key point is the relative magnitudes of the various cross-sections and their sensitivities to $\lambda_{33 i}^{\prime \prime}$. By looking at Fig. 1, one could naively expect the six channels listed in Table I to all scale similarly with $\left(\lambda_{33 i}^{\prime \prime}\right)^{4}$. If this were the case, then the relative magnitudes of the corresponding cross-sections would not to be affected by $\lambda_{33 i}^{\prime \prime}$, and the longer chains would yield smaller cross-sections due to phase space effects as well as to matrix elements suppression by other couplings and intermediate propagators. There is in fact much more to it if one takes into account total widths and branching ratios of the unstable intermediate particles. This section is devoted to an analytical study of these features. To help understand the sensitivity to the RPV coupling we derive the expressions for the cross-sections of the various stop decay channels relying on the narrow width approximation (NWA), see e.g. [69]. It is well-known that the NWA is not always quantitatively reliable. In particular it can fail not only when couplings are large leading to large widths, but also for mass configurations similar to the ones we are considering in this paper, even for small couplings, that is when daughter and parent particles are very close in mass and the effective center of mass energy at the partonic level is of the same order as (twice) the parent particle mass [70-73]. The quantitative analysis in the subsequent Sections will thus not rely on this approximation. Nonetheless, the NWA renders reasonably well the qualitative behavior, providing a physical

\footnotetext{
${ }^{3}$ In such configurations where the decay into gravitinos can be comparable to the RPV decays, one could make use of the very different scaling in $m_{\tilde{t}}$ in the $c \tau$ 's, namely $m_{\tilde{t}}^{4}$ for the RPC-like decay, as compared to $m_{\tilde{t}}^{-5}$ or $m_{\chi^{0}}^{-5}$ for the stop or the neutralino decaying into gravitinos, to extract information from limits on both prompt decays and displaced vertices, see e.g. [44].
} 
understanding of the effects. Moreover in the configurations where the NWA is expected to be valid, a very good quantitative agreement with the numerical simulation based on exact matrix element calculation gives a significant cross-check of the results.

Following the discussion in Section IIIB, the predominant decay chain for the RPVMSSM-LSP is $\chi^{0} \rightarrow \tilde{t}^{*} \bar{t}\left(\tilde{\bar{t}}^{*} t\right) \rightarrow \bar{b} \bar{d} \bar{b}\left(b d_{i} b\right) f_{1} \bar{f}_{1}^{\prime}$. We can thus take, irrespective of the mass hierarchy involving $\tilde{t}$ and $\chi^{+}$:

$$
B R\left(\chi^{0} \rightarrow \tilde{t}^{*} \bar{t}\left(\tilde{\bar{t}}^{*} t\right) \rightarrow \bar{b} \bar{d}_{i} \bar{b}\left(b d_{i} b\right) f_{1} \bar{f}_{1}^{\prime}\right) \approx 1
$$

To be specific we first derive the various expressions under the assumptions $\lambda_{332}^{\prime \prime} \neq 0, \lambda_{331}^{\prime \prime}=$ 0 , and $d_{i}=s$ (i.e. $i=2$ ). Defining

$$
\begin{aligned}
\Gamma_{\tilde{t}-\mathrm{RPV}} & \equiv \Gamma(\tilde{t} \rightarrow \bar{b} \bar{s}) \\
\Gamma_{\chi \mathrm{RPV}} & \equiv \Gamma(\tilde{t} \rightarrow \bar{b} \bar{s} \bar{b} b) \\
\Gamma_{\mathrm{RPC}-\mathrm{like}} & \equiv \Gamma\left(\tilde{t} \rightarrow \bar{b} \bar{s} \bar{b}(b s b) f_{1} \bar{f}_{1}^{\prime} b f \bar{f}^{\prime}\right),
\end{aligned}
$$

the NWA allows to write,

$$
\begin{aligned}
\Gamma_{\chi \mathrm{RPV}} & \simeq \Gamma\left(\tilde{t} \rightarrow \chi^{+} b\right) \times B R\left(\chi^{+} \rightarrow \bar{b} \bar{s} \bar{b}\right) \\
\Gamma_{\mathrm{RPC}-\mathrm{like}} & \simeq \Gamma\left(\tilde{t} \rightarrow \chi^{+} b\right) \times B R\left(\chi^{+} \rightarrow \bar{b} \bar{s} \bar{b}(b s b) f_{1} \bar{f}_{1}^{\prime} f \bar{f}^{\prime}\right) \\
& \simeq \Gamma\left(\tilde{t} \rightarrow \chi^{0} f^{f^{\prime}} b\right) \times B R\left(\chi^{0} \rightarrow \bar{b} \bar{s} \bar{b}(b s b) f_{1} \bar{f}_{1}^{\prime}\right) \\
& \simeq \Gamma\left(\tilde{t} \rightarrow \chi^{0} f \bar{f}^{\prime} b\right)
\end{aligned}
$$

where we made use of Eq. (4.1) when writing Eq. (4.7). Moreover, the fact that $\chi^{+}$decays with branching ratio $\simeq 1$ into $\bar{b} \bar{s} \bar{b}$ and $\bar{b} \bar{s} \bar{b}(b s b) f_{1} \bar{f}_{1}^{\prime} f \bar{f}^{\prime}$ leads through Eqs. $(4.5,4.6)$ to

$$
\Gamma_{\chi-\mathrm{RPV}}+\Gamma_{\mathrm{RPC}-\text { like }} \simeq \Gamma\left(\tilde{t} \rightarrow \chi^{+} b\right) \simeq \text { " } \lambda_{332}^{\prime \prime} \text {-independent" }
$$

A residual sensitivity to $\lambda_{332}^{\prime \prime}$ in $\Gamma_{\chi-\mathrm{RPV}}+\Gamma_{\mathrm{RPC}-\text { like }}$ would still come from loop contributions to the stop mass itself that enters $\Gamma\left(\tilde{t} \rightarrow \chi^{+} b\right)$. However this higher order effect is essentially screened for the range $\lambda_{332}^{\prime \prime} \lesssim 0.1$ under consideration. Therefore, the only significant dependence on the RPV coupling in the stop total width ${ }^{4}, \Gamma_{\tilde{t} \text {-RPV }}+\Gamma_{\chi-R P V}+\Gamma_{\mathrm{RPC}-\text { like }}$, originates from the two body stop decay which can be parametrized as follows,

$$
\Gamma_{\tilde{t}-\mathrm{RPV}}=\left(\lambda_{332}^{\prime \prime}\right)^{2} \times \Gamma_{1}(\tilde{t} \rightarrow \bar{b} \bar{s})
$$

$\overline{4}$ neglecting flavor violating transitions such as $\tilde{t} \rightarrow \chi^{+} s$ and the decay channels $\tilde{t} \rightarrow \chi^{0} t^{*} \rightarrow \chi^{0} b f_{1} \bar{f}_{1}^{\prime}$ or $\tilde{t} \rightarrow h^{0} \tilde{t}^{*} \rightarrow h^{0} \bar{b} \bar{s}$ as noted in Section III B. 
with the notation

$$
\Gamma_{1} \equiv \Gamma_{\mid \lambda_{332}^{\prime \prime}=1}
$$

We now show that the longest decay chain width $\Gamma_{\text {RPC-like }}$ is not always negligible with respect to $\Gamma_{\chi-\mathrm{RPV}}$ or $\Gamma_{\tilde{t}-\mathrm{RPV}}$ and can even overpower these. The relative magnitude of $\Gamma_{\mathrm{RPC}-\text { like }}$ and $\Gamma_{\chi \mathrm{RPV}}$ is controlled by that of $B R\left(\chi^{+} \rightarrow \bar{b} \bar{s} \bar{b}\right)$ and $B R\left(\chi^{+} \rightarrow \bar{b} \bar{s} \bar{b}(b s b) f_{1} \bar{f}_{1}^{\prime} f \bar{f}^{\prime}\right)$ through Eqs. $(4.5,4.6)$, where the relative magnitude of the latter branching ratios depends on the value of $\lambda_{332}^{\prime \prime}$. Indeed, on the one hand the NWA and Eq. (4.1) imply that

$$
\Gamma\left(\chi^{+} \rightarrow \bar{b} \bar{s} \bar{b}(b s b) f_{1} \bar{f}_{1}^{\prime} f \bar{f}^{\prime}\right)=\Gamma\left(\chi^{+} \rightarrow \chi^{0} f \bar{f}^{\prime}\right) \times B R\left(\chi^{0} \rightarrow \bar{b} \bar{s} \bar{b}(b s b) f_{1} \bar{f}_{1}^{\prime}\right) \simeq \Gamma\left(\chi^{+} \rightarrow \chi^{0} f \bar{f}^{\prime}\right)
$$

showing that $\Gamma\left(\chi^{+} \rightarrow \bar{b} \bar{s} \bar{b}(b s b) f_{1} \bar{f}_{1}^{\prime} f \bar{f}^{\prime}\right)$ is essentially $\lambda_{332}^{\prime \prime}$ independent and is identical to the $\chi^{+}$width of the RPC case $\Gamma\left(\chi^{+} \rightarrow \chi^{0} f \bar{f}^{\prime}\right)$. On the other hand, since the stop is off-shell in the decay $\chi^{+} \rightarrow \bar{b} \bar{s} \bar{b}$, obviously the corresponding width scales with $\left(\lambda_{332}^{\prime \prime}\right)^{2}$,

$$
\Gamma\left(\chi^{+} \rightarrow \bar{b} \bar{s} \bar{b}\right)=\left(\lambda_{332}^{\prime \prime}\right)^{2} \times \Gamma_{1}\left(\chi^{+} \rightarrow \bar{b} \bar{s} \bar{b}\right)
$$

Let us now define the following two ratios,

$$
\begin{aligned}
& r_{1} \equiv \frac{\Gamma_{1}(\tilde{t} \rightarrow \bar{b} \bar{s})}{\Gamma\left(\tilde{t} \rightarrow \chi^{+} b\right)}, \\
& r_{2} \equiv \frac{\Gamma_{1}\left(\chi^{+} \rightarrow \bar{b} \bar{s} \bar{b}\right)}{\Gamma\left(\chi^{+} \rightarrow \bar{b} \bar{s} \bar{b}(b s b) f_{1} \bar{f}_{1}^{\prime} f \bar{f}^{\prime}\right)}=\frac{\Gamma_{1}\left(\chi^{+} \rightarrow \bar{b} \bar{s} \bar{b}\right)}{\Gamma\left(\chi^{+} \rightarrow \chi^{0} f^{\prime} \bar{f}^{\prime}\right)}
\end{aligned}
$$

that are essentially $\lambda_{33 i}^{\prime \prime}$ independent (apart from a very small sensitivity in the loop correction to the stop mass, as noted previously), and determined mainly by the RPC parameters of the MSSM. The dependence of the chargino decay branching ratios on $\lambda_{332}^{\prime \prime}$ follows then easily from Eqs. (4.11, 4.12, 4.14),

$$
\begin{aligned}
B R\left(\chi^{+} \rightarrow \bar{b} \bar{s} \bar{b}\right) & =\frac{r_{2} \times\left(\lambda_{332}^{\prime \prime}\right)^{2}}{1+r_{2} \times\left(\lambda_{332}^{\prime \prime}\right)^{2}}, \\
B R\left(\chi^{+} \rightarrow \bar{b} \bar{s} \bar{b}(b s b) f_{1} \bar{f}_{1}^{\prime} f \bar{f}^{\prime}\right) & =\frac{1}{1+r_{2} \times\left(\lambda_{332}^{\prime \prime}\right)^{2}} .
\end{aligned}
$$

It is clear from these expressions that for sufficiently small $\lambda_{332}^{\prime \prime}$ the $\mathbf{R P C}$-like decay $\chi^{+} \rightarrow$ $\bar{b} \bar{s} \bar{b}(b s b) f_{1} \bar{f}_{1}^{\prime} f \bar{f}^{\prime}$ becomes comparable or even dominates the RPV decay $\chi^{+} \rightarrow \bar{b} \bar{s} \bar{b}$. Upon use of Eqs. $(4.5,4.6,4.9)$ the same conclusion holds for the stop widths: the size of $\lambda_{332}^{\prime \prime}$ controls the relative magnitudes of $\Gamma_{\tilde{t}-\mathrm{RPV}}, \Gamma_{\chi-\mathrm{RPV}}$ and $\Gamma_{\mathrm{RPC}-\text { like }}$, the latter becoming largely dominant for a very small RPV coupling! 
We note in passing that the form of Eq. (4.7) might wrongly suggest that $\Gamma_{\text {RPC-like }}$ is $\lambda_{332}^{\prime \prime}$ independent. In fact the $\lambda_{332}^{\prime \prime}$ dependence in $\Gamma\left(\tilde{t} \rightarrow \chi^{0} f^{\prime} b\right)$ is encoded in the total width of $\chi^{+}$, or equivalently in $B R\left(\chi^{+} \rightarrow \bar{b} \bar{s} \bar{b}(b s b) f_{1} \bar{f}_{1}^{\prime} f \bar{f}^{\prime}\right)$. This should be contrasted with $\Gamma\left(\chi^{+} \rightarrow \bar{b} \bar{s} \bar{b}(b s b) f_{1} \bar{f}_{1}^{\prime} f \bar{f}^{\prime}\right)$ which is independent of $\lambda_{332}^{\prime \prime}$.

Using the above results, it is now straightforward to express the stop decay branching ratios, and the stop pair production and decay cross-sections, in terms of $\lambda_{332}^{\prime \prime}, r_{1}$ and $r_{2}$. Before doing so, we note first that all the above steps remain valid if $\lambda_{332}^{\prime \prime}$ is replaced by $\lambda_{331}^{\prime \prime}$ and the $s$ - replaced by the $d$-quark, but also when both couplings $\lambda_{331}^{\prime \prime}$ and $\lambda_{332}^{\prime \prime}$ are simultaneously non-vanishing. Since the difference between the $d$ - and $s$-quark masses is irrelevant, the ratios $r_{1}$ and $r_{2}$ are essentially unchanged when replacing the $s$ - by a $d$-quark. The general case, summing up the $s$ and $d$ contributions, is thus obtained by simply replacing $\lambda_{332}^{\prime \prime}$ by $\lambda_{33 i}^{\prime \prime}$ with

$$
\lambda_{33 i}^{\prime \prime} \equiv \sqrt{\left(\lambda_{332}^{\prime \prime}\right)^{2}+\left(\lambda_{331}^{\prime \prime}\right)^{2}}
$$

in the above formulae. Putting everything together one finds the following general form for the stop pair production and decay cross-sections:

- $\tilde{\mathbf{t}}-\mathbf{R P V}-\tilde{\mathbf{t}}-\mathbf{R P V} \equiv 2 b 2 j$,

$$
\begin{aligned}
\sigma(2 b 2 j) & \simeq \sigma(p p \rightarrow \tilde{\tilde{t}}) \times B R\left(\tilde{t} \rightarrow \bar{b} \bar{d}_{i}\right) \times B R\left(\overline{\tilde{t}} \rightarrow b d_{i}\right) \\
& \simeq \sigma(p p \rightarrow \tilde{\tilde{t}}) \times \frac{r_{1}^{2} \times\left(\lambda_{33 i}^{\prime \prime}\right)^{4}}{\left(1+r_{1} \times\left(\lambda_{33 i}^{\prime \prime}\right)^{2}\right)^{2}},
\end{aligned}
$$

with

$$
B R\left(\tilde{t} \rightarrow \bar{b} \bar{d}_{i}\right)=\frac{\Gamma_{\tilde{t}-\mathrm{RPV}}}{\Gamma_{\tilde{t}-\mathrm{RPV}}+\Gamma_{\chi-\mathrm{RPV}}+\Gamma_{\mathrm{RPC}-\mathrm{like}}}
$$

- $\tilde{\mathbf{t}}-\mathbf{R P V}-\boldsymbol{\chi}-\mathbf{R P V} \equiv 4 b 2 j$,

$$
\begin{aligned}
& \sigma(4 b 2 j) \simeq \sigma(p p \rightarrow \tilde{t} \bar{t}) \times\left(B R\left(\tilde{t} \rightarrow \bar{b} \bar{d}_{i} \bar{b} b\right) \times B R\left(\overline{\tilde{t}} \rightarrow b d_{i}\right)\right. \\
&\left.+B R\left(\overline{\tilde{t}} \rightarrow b d_{i} b \bar{b}\right) \times B R\left(\tilde{t} \rightarrow \bar{b} \bar{d}_{i}\right)\right) \\
& \simeq 2 \times \sigma(p p \rightarrow \tilde{\tilde{t}}) \times B R\left(\tilde{t} \rightarrow \bar{b} \bar{d}_{i} \bar{b} b\right) \times B R\left(\overline{\tilde{t}} \rightarrow b d_{i}\right) \\
& \simeq \sigma(p p \rightarrow \tilde{\tilde{t}}) \times \frac{2 r_{1} r_{2} \times\left(\lambda_{33 i}^{\prime \prime}\right)^{4}}{\left(1+r_{1} \times\left(\lambda_{33 i}^{\prime \prime}\right)^{2}\right)^{2}\left(1+r_{2} \times\left(\lambda_{33 i}^{\prime \prime}\right)^{2}\right)}
\end{aligned}
$$

with

$$
B R(\tilde{t} \rightarrow \bar{b} \bar{s} \bar{b} b)=\frac{\Gamma_{\chi-\mathrm{RPV}}}{\Gamma_{\tilde{t}-\mathrm{RPV}}+\Gamma_{\chi \mathrm{RPV}}+\Gamma_{\mathrm{RPC}-\text { like }}} .
$$


- $\chi-\mathrm{RPV}-\chi-\mathrm{RPV} \equiv 6 b 2 j$,

$$
\begin{aligned}
\sigma(6 b 2 j) & \simeq \sigma(p p \rightarrow \tilde{\tilde{t}}) \times B R\left(\tilde{t} \rightarrow \bar{b} \bar{d}_{i} \bar{b} b\right) \times B R\left(\overline{\tilde{t}} \rightarrow b d_{i} b \bar{b}\right) \\
& \simeq \sigma(p p \rightarrow \tilde{\tilde{t}}) \times \frac{r_{2}^{2} \times\left(\lambda_{33 i}^{\prime \prime}\right)^{4}}{\left(1+r_{1} \times\left(\lambda_{33 i}^{\prime \prime}\right)^{2}\right)^{2}\left(1+r_{2} \times\left(\lambda_{33 i}^{\prime \prime}\right)^{2}\right)^{2}} .
\end{aligned}
$$

- RPC-like- $\tilde{\mathbf{t}}-\mathbf{R P V} \equiv 1 t 3 b 2 j$,

$$
\begin{aligned}
& \sigma(1 t 3 b 2 j) \simeq \sigma(p p \rightarrow \tilde{t} \bar{t}) \times( B R\left(\tilde{t} \rightarrow \bar{b} \bar{d}_{i} \bar{b}\left(b d_{i} b\right) f_{1} \bar{f}_{1}^{\prime} b f \bar{f}^{\prime}\right) \times B R\left(\overline{\tilde{t}} \rightarrow b d_{i}\right) \\
&\left.+B R(\tilde{t} \rightarrow j \bar{b}) \times B R\left(\overline{\tilde{t}} \rightarrow b d_{i} b\left(\bar{b} \bar{d}_{i} \bar{b}\right) \bar{f}_{1} f_{1}^{\prime} \bar{b} \bar{f} f^{\prime}\right)\right) \\
& \simeq 2 \times \sigma(p p \rightarrow \tilde{t} \bar{t}) \times B R\left(\tilde{t} \rightarrow \bar{b} \bar{d}_{i} \bar{b}\left(b d_{i} b\right) f_{1} \bar{f}_{1}^{\prime} b f \bar{f}^{\prime}\right) \times B R\left(\overline{\tilde{t}} \rightarrow b d_{i}\right) \\
& \simeq \sigma(p p \rightarrow \tilde{t} \bar{t}) \times \frac{2 r_{1} \times\left(\lambda_{33 i}^{\prime \prime}\right)^{2}}{\left(1+r_{1} \times\left(\lambda_{33 i}^{\prime \prime}\right)^{2}\right)^{2}\left(1+r_{2} \times\left(\lambda_{33 i}^{\prime \prime}\right)^{2}\right)}
\end{aligned}
$$

with

$$
B R\left(\tilde{t} \rightarrow \bar{b} \bar{d}_{i} \bar{b}\left(b d_{i} b\right) f_{1} \bar{f}_{1}^{\prime} b f \bar{f}^{\prime}\right)=\frac{\Gamma_{\mathrm{RPC}-\text { like }}}{\Gamma_{\tilde{t} \text { RPV }}+\Gamma_{\chi \mathrm{RPV}}+\Gamma_{\mathrm{RPC}-\text { like }}} .
$$

- RPC-like- $\chi-\mathbf{R P V} \equiv 1 t 5 b 2 j$,

$$
\begin{aligned}
& \sigma(1 t 5 b 2 j) \simeq \sigma(p p \rightarrow \tilde{\tilde{t}}) \times( B R\left(\tilde{t} \rightarrow \bar{b} \bar{d}_{i} \bar{b}\left(b d_{i} b\right) f_{1} \bar{f}_{1}^{\prime} b f \bar{f}^{\prime}\right) \times B R\left(\overline{\tilde{t}} \rightarrow b d_{i} b \bar{b}\right) \\
&\left.+B R\left(\tilde{t} \rightarrow \bar{b} \overline{d_{i}} \bar{b} b\right) \times B R\left(\overline{\tilde{t}} \rightarrow b d_{i} b\left(\bar{b} \bar{d}_{i} \bar{b}\right) \bar{f}_{1} f_{1}^{\prime} \bar{b} \bar{f} f^{\prime}\right)\right) \\
& \simeq 2 \times \sigma(p p \rightarrow \tilde{\bar{t}}) \times B R\left(\tilde{t} \rightarrow \bar{b} \bar{d}_{i} \bar{b}\left(b d_{i} b\right) f_{1} \bar{f}_{1}^{\prime} b f \bar{f}^{\prime}\right) \times B R\left(\overline{\tilde{t}} \rightarrow b d_{i} b \bar{b}\right) \\
& \simeq \sigma(p p \rightarrow \tilde{\tilde{t}}) \times \frac{2 r_{2} \times\left(\lambda_{33 i}^{\prime \prime}\right)^{2}}{\left(1+r_{1} \times\left(\lambda_{33 i}^{\prime \prime}\right)^{2}\right)^{2}\left(1+r_{2} \times\left(\lambda_{33 i}^{\prime \prime}\right)^{2}\right)^{2}}
\end{aligned}
$$

- RPC-like-RPC-like $\equiv 2 t 4 b 2 j$,

$$
\begin{aligned}
\sigma(2 t 4 b 2 j) & \simeq \sigma(p p \rightarrow \tilde{\tilde{t}}) \times B R\left(\tilde{t} \rightarrow \bar{b} \bar{d}_{i} \bar{b}\left(b d_{i} b\right) f_{1} \bar{f}_{1}^{\prime} b f_{f^{\prime}}\right) \times B R\left(\overline{\tilde{t}} \rightarrow b d_{i} b\left(\bar{b} \bar{d}_{i} \bar{b}\right) \bar{f}_{1} f_{1}^{\prime} \bar{b} \bar{f} f^{\prime}\right) \\
& \simeq \sigma(p p \rightarrow \tilde{\tilde{t}}) \times \frac{1}{\left(1+r_{1} \times\left(\lambda_{33 i}^{\prime \prime}\right)^{2}\right)^{2}\left(1+r_{2} \times\left(\lambda_{33 i}^{\prime \prime}\right)^{2}\right)^{2}} .
\end{aligned}
$$

We have replaced $s$ by $d_{i}$ in the above expressions to stress the fact that these are valid either for the case of $s$ alone, or for the case of $d$ alone, or else for the sum of the two, depending on the values of $\lambda_{331}^{\prime \prime}, \lambda_{332}^{\prime \prime}$ in Eq. (4.17).

The analytical form of Eqs. $(4.18$ - 4.23) illustrate clearly the deviation from the naive expectation that all cross-sections would scale with $\left(\lambda_{33 i}^{\prime \prime}\right)^{4}$. One sees that such scaling is generically modified by the RPC-like component. Moreover, even for the $\tilde{\mathbf{t}}-\mathbf{R P V}$ and $\boldsymbol{\chi}$ RPV contributions different final state cross-sections can have various sensitivities to $\lambda_{332}^{\prime \prime}$ depending on the following possible regimes: 


$$
r_{a} \ll\left(\lambda_{33 i}^{\prime \prime}\right)^{-2}, r_{a} \sim\left(\lambda_{33 i}^{\prime \prime}\right)^{-2}, r_{a} \gg\left(\lambda_{33 i}^{\prime \prime}\right)^{-2}, \quad(a=1,2)
$$

These regimes are triggered by the interplay between the RPV and RPC sectors. For instance the magnitude of $r_{1}$ is controlled by the degree of mass degeneracy between the stop and the chargino. Similarly, the degeneracy between the chargino and neutralino masses implies typically a large $r_{2}$. Perhaps the most striking feature that comes out of the NWA expressions is that the variation of $\lambda_{33 i}^{\prime \prime}$ over several orders of magnitude, within the range given in Eq. (3.1), triggers the dominance of very different final states without reducing the total cross-sections. In particular, while the $\tilde{\mathbf{t}}-\mathbf{R P V}-\tilde{\mathbf{t}}-\mathbf{R P V}$ clearly dominates for relatively large values of $\lambda_{33 i}^{\prime \prime}$, the $\mathbf{R P C}$-like-RPC-like becomes dominant for very small values of this coupling. Furthermore, one can easily determine from Eqs. (4.18, 4.19,4.20, $4.22,4.23)$ the scaling relations

$$
\begin{gathered}
\frac{\sigma(2 b 2 j) \cdot \sigma(6 b 2 j)}{[\sigma(4 b 2 j)]^{2}}=\frac{1}{4}, \\
\frac{\sigma(6 b 2 j) \cdot \sigma(2 t 4 b 2 j)}{[\sigma(1 t 5 b 2 j)]^{2}}=\frac{1}{4} .
\end{gathered}
$$

We refer to these two scaling relations respectively as b-SR and t-SR, where the first one involves shorter decay chains with no top-quark final states and the second longer decay chains with top-quark final states. These scaling relations lead also to

$$
\frac{\sigma(2 t 4 b 2 j)}{\sigma(2 b 2 j)}=\left(\frac{\sigma(1 t 5 b 2 j)}{\sigma(4 b 2 j)}\right)^{2} \text {. }
$$

To summarize, we derived in this Section analytical expressions for the cross-sections with all possible stop decay final states, in a form that untangles the dependence on the RPV $\lambda_{33 i}^{\prime \prime}$ coupling from that on the MSSM mass spectrum and RPC couplings encoded in the $r_{a}$ ratios Eqs. $(4.13,4.14)$. Moreover these expressions imply scaling relations among the cross-sections independently of the couplings and masses. Given the complexity of the long chain decays, these analytical results will prove very useful, even though established within the approximation of narrow width, when interpreting the results and assessing the validity of the exact matrix element numerical computation in Section VI. 


\section{BENCHMARK POINTS AND CONSTRAINTS}

In order to estimate the cross-sections for the processes of interest, we interfaced several software packages as discussed in the following. Firstly we used the SARAH [74] Mathematica [75] package to generate model files in UFO format compatible with the MadGraph5_AMC@NLO [76] Monte Carlo generator. Then we used SARAh to implement the MSSM trilinear RPV model in SPHENo [77] so as to calculate the entire SUSY mass spectrum and couplings. We adopted a bottom-bottom approach, where the values of the supersymmetric and soft SUSY breaking parameters are provided directly at the electroweak scale. This approach has the benefit of being simple without sacrificing the typical supersymmetric correlations among various low energy states masses and couplings, and of being model-independent in view of our present ignorance of how supersymmetry is realized at high scales.

Using the low scale MSSM option of the SPHENo code, we performed a scan over the SUSY input parameters to determine benchmark points that are consistent with our spectrum assumptions discussed in Sections II C and III, as well as with constraints from the available physical observables. We generated several mass spectra in different regions of the relevant MSSM parameter space, fixing the EWSB scale to $Q_{E W S B}=1 \mathrm{TeV}$ and including 1-loop corrections to all SUSY particle masses and 2-loop corrections to the lightest CP-even Higgs mass. For each given parameter point we used HigasBounds [78, 79] and HiggsSignals [80] to confront the Higgs sector computed by SPHENo with existing measurements and exclusion limits. Moreover we accounted for the low energy flavor constraints coming from the recent measurements of $B^{0}$ decaying into a pair of muons [81-83]. For given values of the soft SUSY breaking parameters in the stop and gaugino sectors satisfying these constraints, a further scan over the $\mu$ parameter was performed such that the lighter chargino and neutralinos remain Higgsino-like and the resulting masses reproduce the hierarchy given by Eqs. $(2.11$ - 2.13). For the remainder of this paper we choose two benchmark sets of input parameters as given in Table II, corresponding to two stop mass values $m_{\tilde{t}}=600 \mathrm{GeV}$ and $1 \mathrm{TeV}$. The values we take in Table II should be understood as given at the EWSB scale. Note that we have put to zero several of these parameters (see last line of Table II), in particular the off-diagonal components in flavor space of soft masses keeping up with our MFV assumption, and the soft SUSY breaking trilinear couplings $T_{33 i}^{\prime \prime}$ 
associated with $\lambda_{33 i}^{\prime \prime}$ as they involve only scalar states and thus would not contribute to our study at leading order.

The large mass splitting between the two stop states in accordance with assumption (ii) of Sec. II C is achieved through the large numerical difference between $\left(m_{\tilde{Q}}\right)_{33}$ and $\left(m_{\tilde{U}}\right)_{33}$ rather than through a large off-diagonal component of the mass matrix. The mixing between the light and heavy stops is thus very small, therefore the lighter stop, essentially righthanded, has its baryon number violating RPV decay controlled mainly by the magnitude of $\lambda_{33 i}^{\prime \prime}$. Note also that the values of $m_{\tilde{t}}$, respectively $600 \mathrm{GeV}$ and $1 \mathrm{TeV}$ in the two benchmark scenarios still vary slightly by about $-0.5 \%+1.5 \%$ around the central value due on one hand to the sensitivity to $\mu$ through the mixing in the stop sector, though suppressed by the moderately large value of $\tan \beta$, and on the other hand to the sensitivity to $\lambda_{33 i}^{\prime \prime}$ through loop corrections [56].

Including 1- and 2-loop corrections from the RPC sector, the lighter CP-even Higgs mass remains essentially at $125 \mathrm{GeV}$, as extra 1-loop corrections from the RPV sector [84] which have also been included, are negligible in the scanned $\lambda_{33 i}^{\prime \prime}$ range given in Eq. (3.1). The small variation in the mass splitting among the light chargino and neutralinos is a residual effect of the small $\mu / M_{1}$ and $\mu / M_{2}$ ratios as already noted in Sec. III. All other states are very heavy (between 1.5 and $3 \mathrm{TeV}$ ) and do not affect our study. Since we rely on the low scale MSSM option the renormalization group running of couplings and masses involves only the range between $m_{Z}$ and the EWSB scale. This allows to treat consistently the gauge and Yukawa couplings extracted at the $m_{Z}$ scale and the input SUSY parameters $\mu, \tan \beta$ and the (tree-level) CP-odd neutral Higgs mass $m_{A}$ defined at the EWSB scale. In particular we make no theoretical assumptions relating the RPV-MSSM parameters at very high scales that would have induced correlations at low scales through the renormalization group evolution. In this context assumption (i) of Sec. II C with values in the range defined in Eq. (3.1) should be viewed as defined at the EWSB scale. The running of $\lambda_{33 i}^{\prime \prime}$ from the EWSB to the $m_{\tilde{t}}$ or $m_{\chi^{+}}$scales where the various stop decay channels are evaluated, remains very small and it is neglected in our study. Note however, that $\lambda_{332}^{\prime \prime}$ affects the running of the top-quark Yukawa coupling between the EWSB scale and $m_{Z}$. Similarly, there are no high scale assumptions about the soft SUSY breaking masses and trilinear couplings. 


\begin{tabular}{|c|c|c|}
\hline Benchmark points & 1 & 2 \\
\hline $\tan \beta$ & \multicolumn{2}{|c|}{10} \\
\hline$M_{1}$ & \multicolumn{2}{|c|}{$2.5 \mathrm{TeV}$} \\
\hline$M_{2}$ & \multicolumn{2}{|c|}{$1.5 \mathrm{TeV}$} \\
\hline$M_{3}$ & \multicolumn{2}{|c|}{$1.7 \mathrm{TeV}$} \\
\hline$\left(m_{\tilde{Q}}\right)_{33}$ & \multicolumn{2}{|c|}{$2 \mathrm{TeV}$} \\
\hline$\left(m_{\tilde{U}}\right)_{33}$ & $570 \mathrm{GeV}$ & $964 \mathrm{GeV}$ \\
\hline$\left(m_{\tilde{D}}\right)_{33}=\left(m_{\tilde{U}}\right)_{i i}=\left(m_{\tilde{D}}\right)_{i i}=\left(m_{\tilde{E}}\right)_{i i}=\left(m_{\tilde{Q}}\right)_{i i}=\left(m_{\tilde{L}}\right)_{i i}, i=1,2$ & \multicolumn{2}{|c|}{$3 \mathrm{TeV}$} \\
\hline$\left(T^{u}\right)_{33}$ & $-2100 \mathrm{GeV}$ & $-2150 \mathrm{GeV}$ \\
\hline$m_{A}$ & \multicolumn{2}{|c|}{$2.5 \mathrm{TeV}$} \\
\hline$\mu$ & $400-650 \mathrm{GeV}$ & 750-1000 GeV \\
\hline$\lambda_{33 i}^{\prime \prime} \equiv \sqrt{\left(\lambda_{332}^{\prime \prime}\right)^{2}+\left(\lambda_{331}^{\prime \prime}\right)^{2}}$ & \multicolumn{2}{|c|}{$10^{-7}-10^{-1}$} \\
\hline$T^{l}, T^{d},\left(T^{u}\right)_{i j},\left(m_{\tilde{Q}, \tilde{U}, \tilde{D}, \tilde{L}, \tilde{E}}\right)_{i j}, T_{33 i}^{\prime \prime}, i \neq j=1,2,3,\left(T^{u}\right)_{i i}, i=1,2$ & \multicolumn{2}{|c|}{0} \\
\hline
\end{tabular}

TABLE II: Two lists of benchmark SUSY parameters defined at the low scale $Q_{E W S B}^{2}=1 \mathrm{TeV}^{2}$ taken as input for SPHENO. All other non-listed supersymmetric or soft SUSY breaking parameters are either computed from the input, such as $m_{H_{1,2}}^{2}$, or irrelevant to the present study, such as $\lambda_{i j k}, \lambda_{i j k}^{\prime}, \mu_{i}, T_{i j k}, T_{i j k}^{\prime}, B_{i}, \tilde{m}_{1 i}$ for all three generations, and $T_{i j k}^{\prime \prime}$ for $i, j=1,2$. We also take $m_{b}\left(m_{b}\right)_{\overline{\mathrm{MS}}}=4.18 \mathrm{GeV}$ and $m_{t}($ pole $)=173.5 \mathrm{GeV}$. See [77] for the values of the other SM input parameters. 


\begin{tabular}{|c|c|c|}
\hline Benchmark points & 1 & 2 \\
\hline$m_{\tilde{t}}$ & $\sim 600 \mathrm{GeV}$ & $\sim 1 \mathrm{TeV}$ \\
\hline$m_{\chi^{+}}$ & $\sim 400-650 \mathrm{GeV}$ & $\sim 750-1000 \mathrm{GeV}$ \\
\hline$m_{\chi^{+}}-m_{\chi^{0}}$ & \multicolumn{2}{|c|}{$\sim 1.5-2.5 \mathrm{GeV}$} \\
\hline$m_{\tilde{t}}-m_{\chi^{+}}$ & $\sim-45-200 \mathrm{GeV}$ & $\sim 1-245 \mathrm{GeV}$ \\
\hline$m_{\chi_{2}^{0}}-m_{\chi^{+}}$ & \multicolumn{2}{|c|}{$\sim 4-5 \mathrm{GeV}$} \\
\hline$m_{\chi_{3}^{0}} \sim m_{\chi_{2}^{+}}, m_{\chi_{4}^{0}}$ & \multicolumn{2}{|c|}{$\sim 1.5 \mathrm{TeV}, \sim 2.5 \mathrm{TeV}$} \\
\hline$m_{h^{0}}$ & \multicolumn{2}{|c|}{$\sim 125 \mathrm{GeV}$} \\
\hline$m_{A} \approx m_{H^{0}} \approx m_{H^{ \pm}}$ & \multicolumn{2}{|c|}{$\sim 2.5 \mathrm{TeV}$} \\
\hline$M_{\tilde{g}}$ & \multicolumn{2}{|c|}{$\sim 1.87 \mathrm{TeV}$} \\
\hline$M_{\tilde{t} 2} \approx M_{\tilde{b} 1}$ & \multicolumn{2}{|c|}{$\sim 2 \mathrm{TeV}$} \\
\hline$M_{\tilde{b} 2} \approx M_{\tilde{u} 1,2} \approx M_{\tilde{d} 1,2}$ & \multicolumn{2}{|c|}{$\sim 3 \mathrm{TeV}$} \\
\hline$M_{\tilde{l} 1,2}, M_{\tilde{\nu} 1,2}$ & \multicolumn{2}{|c|}{$\sim 3 \mathrm{TeV}$} \\
\hline$(g-2)_{\mu}^{\mathrm{SUSY}}$ & $3-3.3 \times 10^{-11}$ & $3.2-3.3 \times 10^{-11}$ \\
\hline$\delta \rho^{\mathrm{SUSY}}$ & $5.7-5.9 \times 10^{-5}$ & $\sim 5.5 \times 10^{-5}$ \\
\hline$B R\left(B \rightarrow X_{s} \gamma\right) / B R\left(B \rightarrow X_{s} \gamma\right)^{S M}$ & $0.89-0.92$ & $0.95-0.96$ \\
\hline$B R\left(B_{s}^{0} \rightarrow \mu \mu\right)$ & $3.36-3.39 \times 10^{-9}$ & $3.38-3.40 \times 10^{-9}$ \\
\hline$B R\left(B_{d}^{0} \rightarrow \mu \mu\right)$ & $1.08-1.09 \times 10^{-10}$ & $\sim 1.09 \times 10^{-10}$ \\
\hline
\end{tabular}

TABLE III: Two lists of benchmark observables generated with SPHENO corresponding to the input of Table II and taken as input for MAdGraph5_AMC@NLO. Pole masses are evaluated at one-loop order except for the lightest CP-even Higgs which includes the 2-loop corrections. 


\section{Low energy constraints}

A large number of low energy and precision observables can be very sensitive to BSM physics. Among these, the LEP/SLC electroweak precision observables, the leptons anomalous magnetic moments and electric dipole moments as well as low energy quark or lepton number violating processes. In Table III we give the values in our two benchmark points of only a few of them. ${ }^{5}$

The anomalous magnetic moment of the muon is a very important test bed for virtual effects from BSM physics as it is one of the most accurately measured quantities in particle physics; for a review see e.g. Ref. [85]. At the one-loop level $(g-2)_{\mu}$ receives contributions from the purely SUSY neutralino/smuon and chargino/muonic-sneutrino RPC sectors. In our benchmark points the smuon sector is very heavy and the chargino/neutralino relatively heavy as well, leading to the small contribution reported in Table III given the chosen moderate value of $\tan \beta$. Other possible one-loop effects from nonzero $\lambda, \lambda^{\prime} \mathrm{RPV}$ couplings, or from CP-violating phases [86], are absent in our scenario. Moreover, two-loop RPC SUSY corrections [87], are not expected to be significant in our case even for a relatively light stop, due to the moderate values of the $\mu$ parameter and $\tan \beta$. The $3.6 \sigma$ discrepancy $\Delta\left(\frac{1}{2}(g-2)_{\mu}\right)=288(63)(49) \times 10^{-11},[11]$, between the experimental measurement and the theoretical SM predictions is thus too large to be accounted for by our benchmark points, leaving open the issue of the uncertainties on the theoretical estimates of the SM hadronic contributions.

Virtual corrections to the $\rho$ parameter originate from the squark and slepton left-handed states. They tend to be suppressed for heavy states as a result of decoupling but can be enhanced by mass splitting between up and down flavors as a result of custodial symmetry breaking [88]. In our benchmark scenario where the lighter stop is mainly right-handed and all other squark and slepton states heavy and almost degenerate, no sizable effects on $\delta \rho$ are expected from these sectors even for a relatively light $\tilde{t}$. The resulting range for $\rho \simeq 1+\delta \rho^{\text {SUSY }}$ obtained in our scan remains consistent within $2 \sigma$ with the experimental value [11].

The $B$-meson radiative inclusive decay $B \rightarrow X_{s} \gamma$ is sensitive to virtual effects from various

\footnotetext{
${ }^{5}$ For more details on the level of accuracy used see [77] and references therein.
} 
sectors of the MSSM associating the charged Higgs to the top quark, the up squarks to the charginos and the down squarks to the neutralinos or to the gluino [89]. Only the $\chi^{+}-\tilde{t}$ loops are sizable in our case as the stop is much lighter than all other squarks and the gluino. Moreover it is mainly right-handed and the chargino higgsino-like, thus further leading to a $\mathcal{O}\left(m_{t} / M_{W}\right)$ enhancement in the amplitude. The charged Higgs yields like-wise suppressed contributions due to its very heavy mass. Taking into account the recent update for the SM theoretical prediction $B R\left(B \rightarrow X_{s} \gamma\right)=(3.36 \pm 0.23) \times 10^{-4}$ [90], our scan remains within $1.2 \sigma$ from the combined experimental value $B R\left(B \rightarrow X_{s} \gamma\right)=(3.43 \pm 0.21 \pm 0.07) \times 10^{-4}[91]$. It is however interesting to note that keeping only the right-handed stop and Higgsinolike contributions, the mass ratio dependence in the loop functions favor, for lighter stops, heavier charginos in order to cope with the $B R\left(B \rightarrow X_{s} \gamma\right)$ constraints. As a consequence a sufficiently light stop would require a reduced mass splitting with respect to the lighter chargino, eventually even forbidding the hierarchy given in Eq. (2.11) and favoring a stop MSSM-LSP. The latter would imply a stop decaying $100 \%$ into $b+$ jet final states, giving support to the model-independence of the present exclusion limits based on this assumption, as long as the ensuing bounds remain low enough. For instance we find that a lower bound of 0.89 on $B R\left(B \rightarrow X_{s} \gamma\right) / B R\left(B \rightarrow X_{s} \gamma\right)^{S M}$ as adopted e.g. in [92] would typically require $m_{\tilde{t}} \gtrsim 400 \mathrm{GeV}$. Still, a more quantitative study is needed as mass degeneracy between the stop and chargino could still be allowed favoring the third regime of Eq. (4.24) and thus final states with $\boldsymbol{\chi}$-RPV or RPC-like components. For instance relaxing the lower bound to $\sim 0.84$ would allow lighter non-LSP stops, e.g. $m_{\tilde{t}} \lesssim 385 \mathrm{GeV}$, with $m_{\chi^{+}} \gtrsim 198 \mathrm{GeV}$.

Finally, regarding the $B^{0}$ decay into a pair of muons, LHCb [81, 82] and CMS [83] have recently reported observation of such decays, with the combined fits leading to $B R\left(B_{s}^{0} \rightarrow\right.$ $\mu \mu)=\left(2.8_{-0.6}^{+0.7}\right) \times 10^{-9}$ and $B R\left(B_{d}^{0} \rightarrow \mu \mu\right)=\left(3.9_{-1.4}^{+1.6}\right) \times 10^{-10}$ that are compatible with the SM at $2 \sigma$-level [93]. Our benchmark numbers are consistent with the updated SM theoretical predictions $B R\left(B_{s}^{0} \rightarrow \mu \mu\right)=(3.65 \pm 0.23) \times 10^{-9}$ and $B R\left(B_{d}^{0} \rightarrow \mu \mu\right)=(1.06 \pm 0.09) \times 10^{-10}$ [94]. 


\section{CROSS-SECTIONS AND UNCERTAINTIES}

Using the spectrum calculator and event generator tools as described in the previous Sections we have computed the total cross-section and decays of a pair of stops in $p p$ collisions at $\sqrt{s}=14 \mathrm{TeV}$ for the two benchmark points given in Table II and the various combinations of final states given in Table I, except for the $1 t 3 b 2 j$ final state since it remains subdominant everywhere in the considered $\lambda_{33 i}^{\prime \prime}$ range. In Figs. 2 and 3 we illustrate the sensitivity to the magnitude of $\lambda_{33 i}^{\prime \prime}$, and in Figs. 4 and 5 the sensitivity to the stop-chargino mass splitting for the two benchmark points. Before commenting these results, we discuss first the various theoretical uncertainties.

\section{A. Theoretical uncertainties}

Besides the BSM uncertainties which cannot be really quantified and are somewhat fixed through the choice of the MSSM parameters, there are other theoretical inputs, whose uncertainties must be taken into account when quoting the expected cross sections for a given process. Since we are interested in the evaluation of the total cross-sections involving the SUSY-QCD process of stop pair production followed by SUSY-EW decays through various short and long chains, we choose to generate the $p p \rightarrow \tilde{\tilde{t}}$ processes at the leading order (LO) accuracy level. SUSY-QCD calculations up to next-to-leading-order (NLO) as well as resummed soft gluons at next-to-leading-logarithmic (NLL) level for the partonic stop pair production cross-section in proton-(anti)proton collisions are well-known, see [95] for a recent appraisal. These calculations contribute to reducing scale uncertainties and typically lead to an increase of the cross-section above LO results [96-98], especially near the partonic stop pair production threshold. On the other hand, Parton Distribution Functions (PDFs) have been recently supplemented by soft gluon threshold resummation at the NLO accuracy [99]. Using these PDFs consistently in conjunction with the resummed partonic matrix element calculations, showed a partial cancellation of the above mentioned threshold effects bringing them closer to the fixed order results. One thus expects the cross-section for heavy stop pair production to be well approximated by fixed order NLO results. Moreover, the latter corrections are in turn expected to be moderate for our benchmark points with very heavy colored SUSY states. In fact comparing for instance the NLO-NLL results 


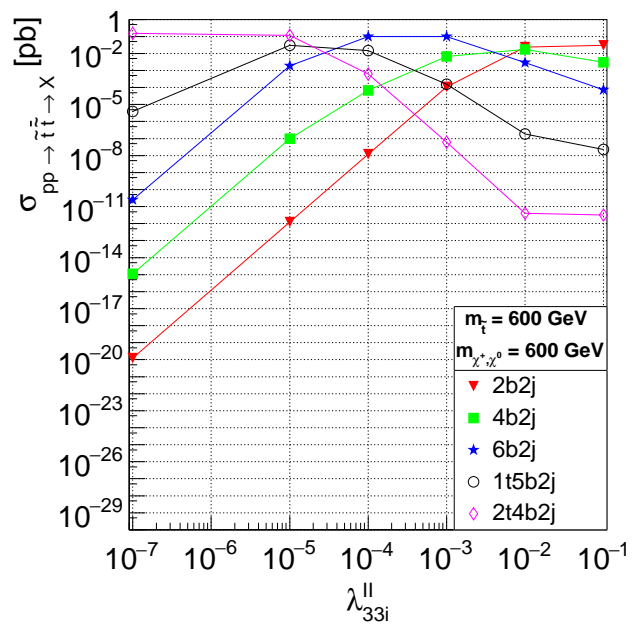

(a)

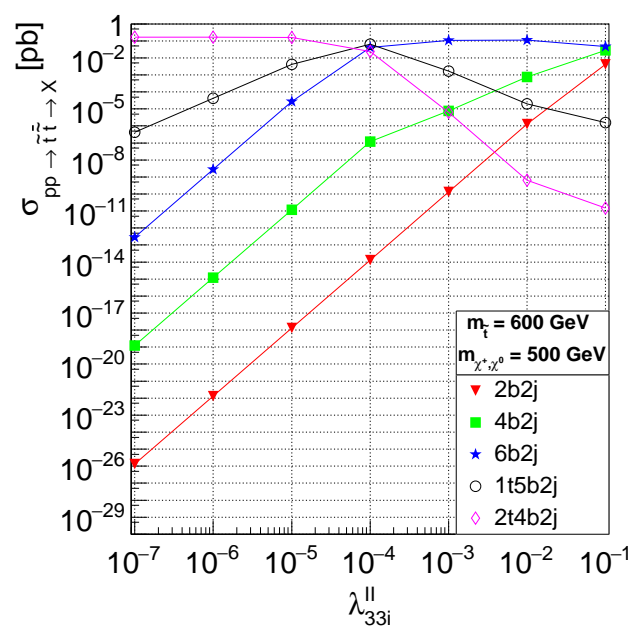

(c)

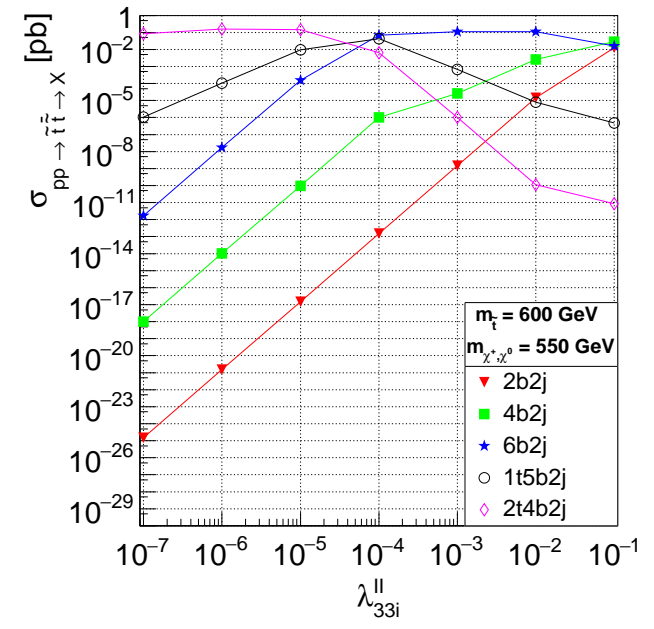

(b)

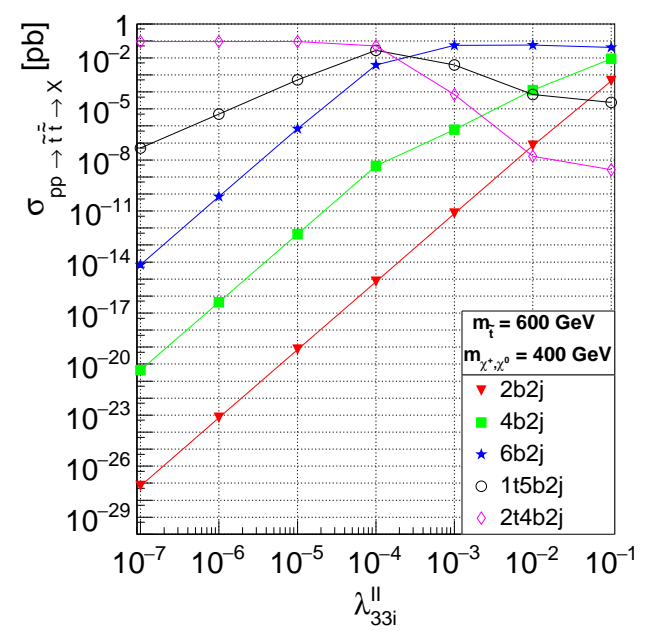

(d)

FIG. 2: Benchmark 1: production cross-section for $\sigma(p p \rightarrow \tilde{\tilde{t}} \rightarrow X)$ at $\sqrt{s}=14 \mathrm{TeV}$, where $X=2 b 2 j$ (red triangles), $4 b 2 j$ (green squares), $6 b 2 j$ (blue stars), $1 t 5 b 2 j$ (black empty circles) and $2 t 4 b 2 j$ (pink diamonds), as a function of $\lambda_{33 i}^{\prime \prime}$ and for $m_{\tilde{t}}-m_{\chi^{+}} \simeq 0 \mathrm{GeV}(\mathrm{a}), 50 \mathrm{GeV}(\mathrm{b}), 100$ $\mathrm{GeV}(\mathrm{c})$ and $200 \mathrm{GeV}(\mathrm{d})$. See Tabs. II and III for the low-energy values of the MSSM parameters.

in the decoupled gluon/squarks limits given in [95] to the LO results we find an increase of the former in excess of $30 \%$ for $m_{\tilde{t}}=600 \mathrm{GeV}$ at $\sqrt{s}=14 \mathrm{TeV}$. However, due to the above mentioned partial cancellation the effect would be smaller for production cross-sections dominated by stops almost at rest when NLL contributions are consistently included also 


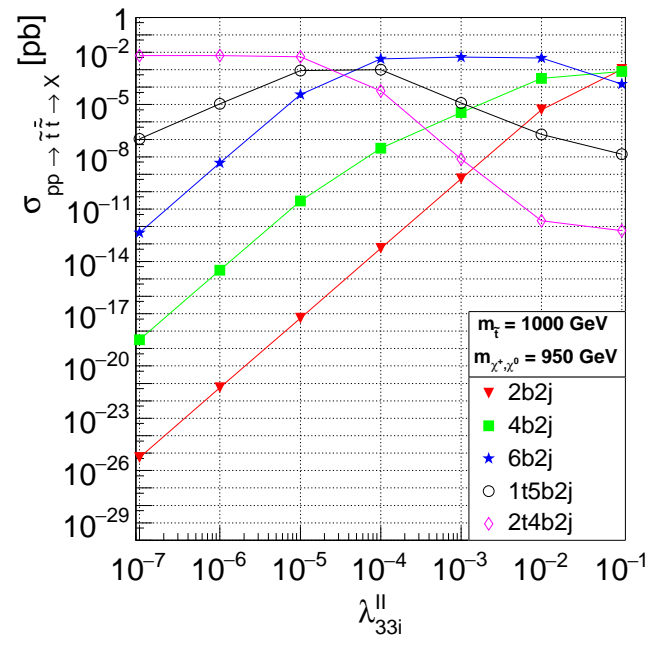

(a)

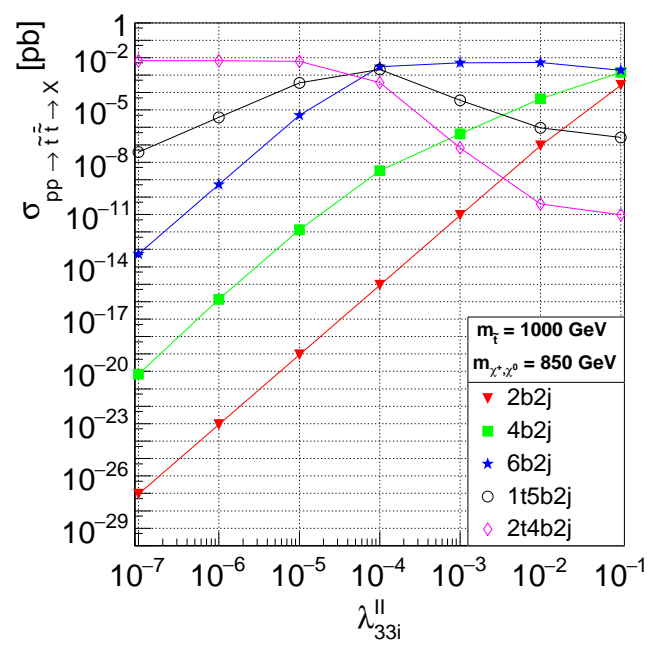

(c)

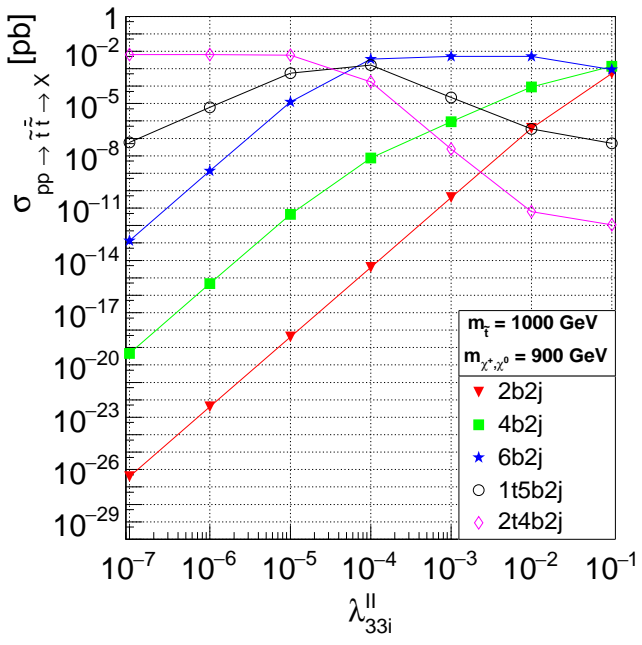

(b)

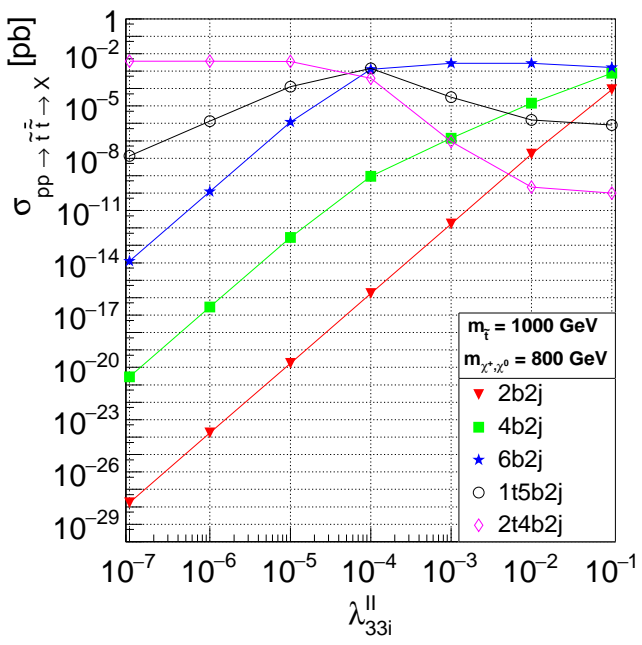

(d)

FIG. 3: Benchmark 2: production cross-section for $\sigma(p p \rightarrow \tilde{\tilde{t}} \rightarrow X)$ at $\sqrt{s}=14 \mathrm{TeV}$, where $X=2 b 2 j$ (red triangles), $4 b 2 j$ (green squares), $6 b 2 j$ (blue stars), $1 t 5 b 2 j$ (black empty circles) and $2 t 4 b 2 j$ (pink diamonds), as a function of $\lambda_{33 i}^{\prime \prime}$ and for $m_{\tilde{t}}-m_{\chi^{+}} \simeq 50 \mathrm{GeV}(\mathrm{a}), 100 \mathrm{GeV}(\mathrm{b})$, $150 \mathrm{GeV}(\mathrm{c})$ and $200 \mathrm{GeV}(\mathrm{d})$. See Tabs. II and III for the low-energy values of the MSSM parameters.

in the PDFs. The difference between NLO and LO production cross-sections would thus be within the uncertainties related to scale variation or to the choice of PDF sets (discussed below). Another reason to stick consistently to LO accuracy for the production cross- 


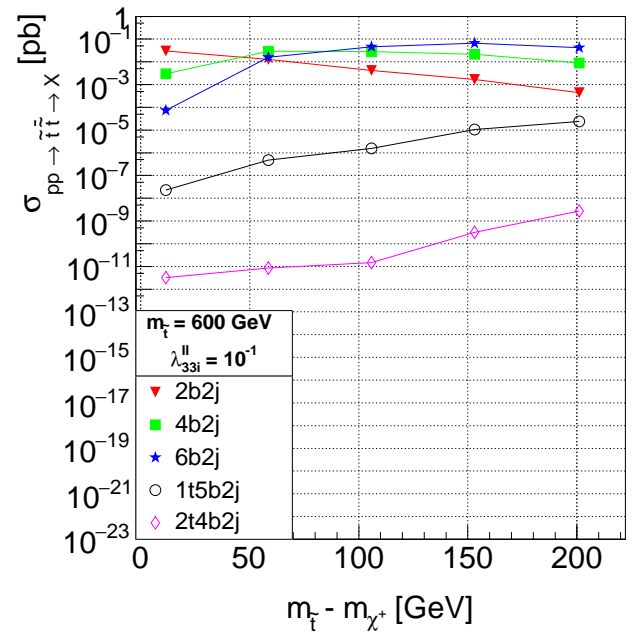

(a)

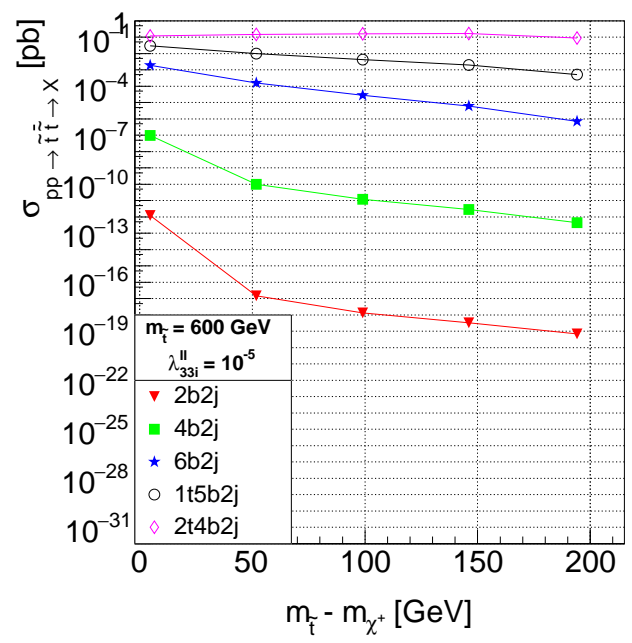

(c)

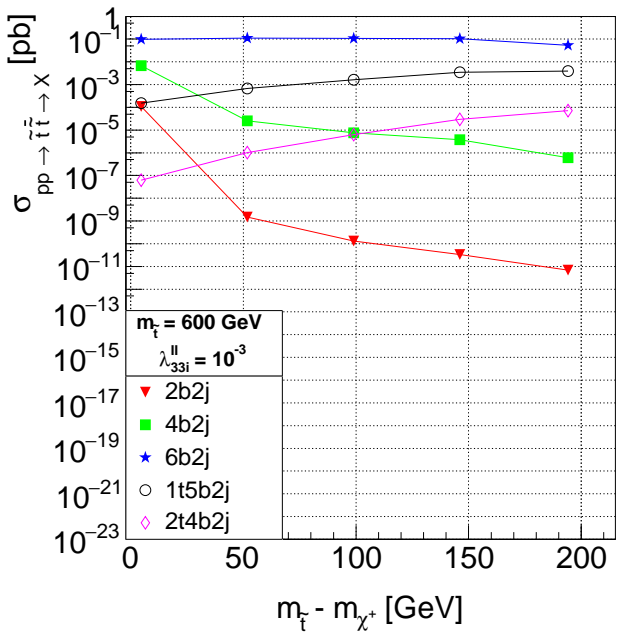

(b)

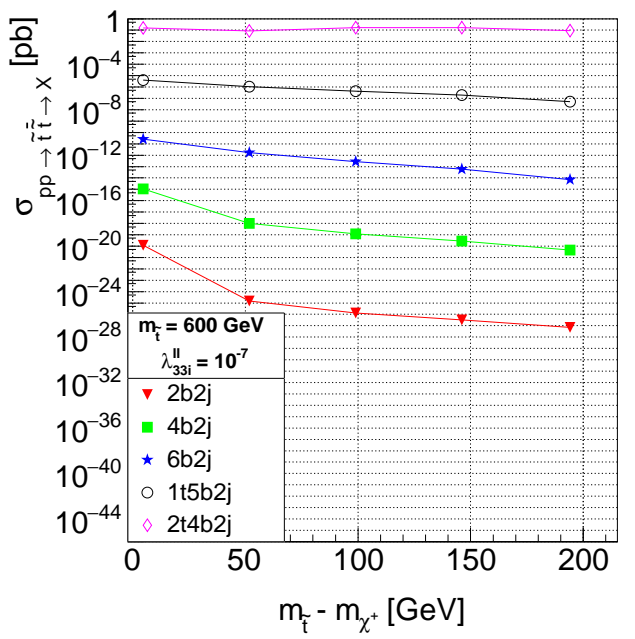

(d)

FIG. 4: Benchmark 1: production cross-section for $\sigma(p p \rightarrow \tilde{\tilde{t}} \rightarrow X)$ at $\sqrt{s}=14 \mathrm{TeV}$, where $X=2 b 2 j$ (red triangles), $4 b 2 j$ (green squares), $6 b 2 j$ (blue stars), $1 t 5 b 2 j$ (black empty circles) and $2 t 4 b 2 j$ (pink diamonds), as a function of $m_{\tilde{t}}-m_{\chi^{+}}$and for $\lambda_{33 i}^{\prime \prime}=10^{-1}$ (a), $10^{-3}$ (b), $10^{-5}$ (c) and $10^{-7}(\mathrm{~d})$. See Tabs. II and III for the low-energy values of the MSSM parameters.

section in the present study, is that the dominant virtual QCD corrections to the stop decay chains are not readily available at the level of matrix element calculations for the considered channels. Moreover, even though some of these corrections could partly cancel in branching ratios, the latter entail the NWA which, as pointed out in Section IV and 


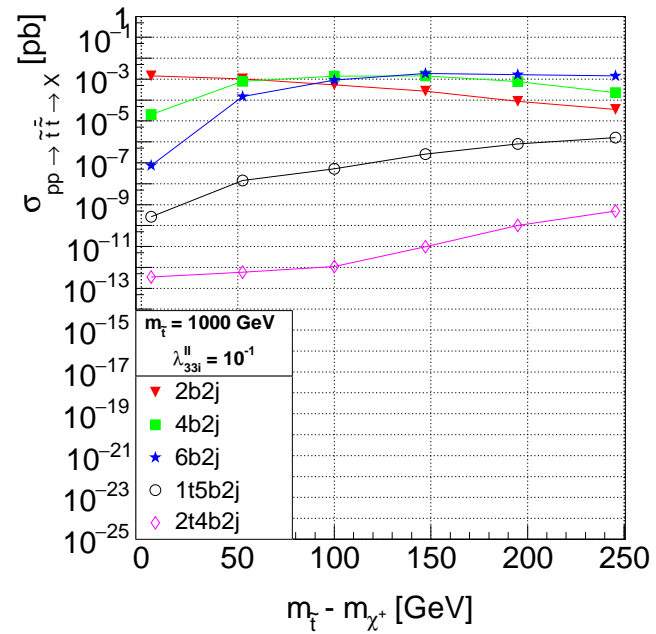

(a)

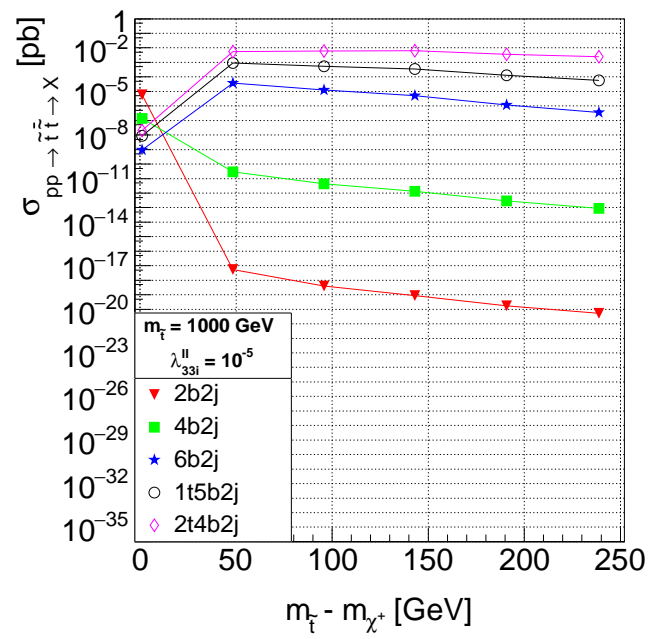

(c)

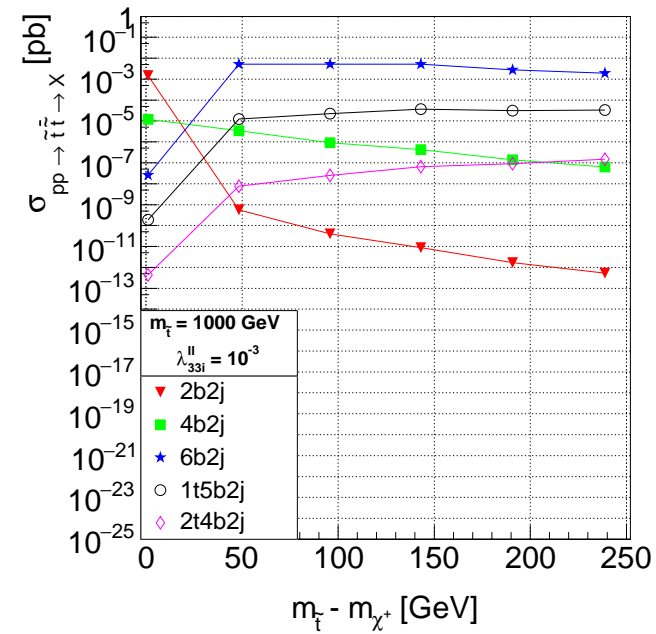

(b)

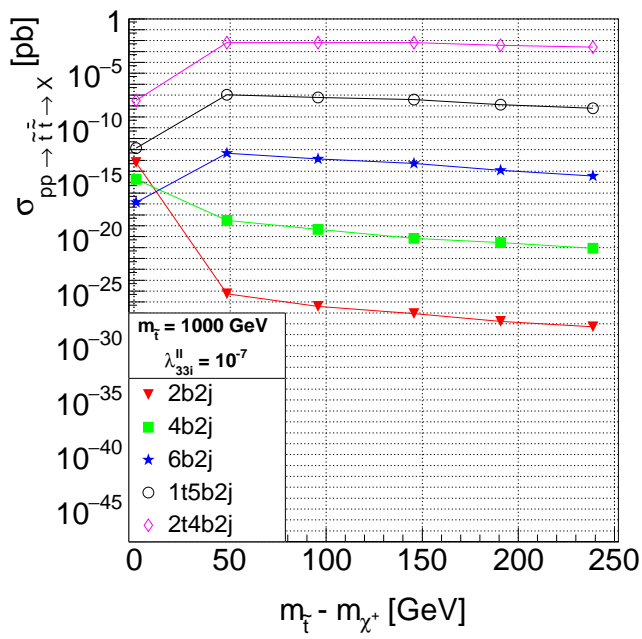

(d)

FIG. 5: Benchmark 2: production cross-section for $\sigma(p p \rightarrow \tilde{t} \bar{t} \rightarrow X)$ at $\sqrt{s}=14 \mathrm{TeV}$, where $X=2 b 2 j$ (red triangles), $4 b 2 j$ (green squares), $6 b 2 j$ (blue stars), $1 t 5 b 2 j$ (black empty circles) and $2 t 4 b 2 j$ (pink diamonds), as a function of $m_{\tilde{t}}-m_{\chi^{+}}$and for $\lambda_{33 i}^{\prime \prime}=10^{-1}$ (a), $10^{-3}$ (b), $10^{-5}$ (c) and $10^{-7}(\mathrm{~d})$. See Tabs. II and III for the low-energy values of the MSSM parameters.

discussed quantitatively in Section VIB, is not always a good approximation to the full matrix element calculations.

We now turn to the uncertainties from the PDFs and from the factorization and renormalization scales, evaluated for the $2 b 2 j$ and $6 b 2 j$ final state processes at the center of mass 
energy of $14 \mathrm{TeV}$ using MADGRAPH5_AMC@NLO.

\section{Systematic uncertainty from scale variation}

In order to evaluate the scale uncertainty, we vary the renormalization and factorization scales independently with respect to the fixed scales central values $\mu_{R}=\mu_{F}=m_{\tilde{t}}$. We choose values within the range $m_{\tilde{t}} / 2<\mu_{R}, \mu_{F}<2 m_{\tilde{t}}$. The computation is performed using the NNPDF23LO1 set [100] for three different stop mass points corresponding to $m_{\tilde{t}}=600$, 800 and $1000 \mathrm{GeV}$, three different stop-chargino mass splitting equal to 50, 100 and 150 $\mathrm{GeV}$ and three different values of the coupling $\lambda_{33 i}^{\prime \prime}=10^{-1}, 10^{-3}$ and $10^{-6}$. At a given $m_{\tilde{t}}$, we take the scale uncertainty to be the largest difference in cross section relative to the central value. We note that the fractional scale uncertainty for both $2 b 2 j$ and $6 b 2 j$ processes is approximately $\begin{aligned} & -25 \% \\ & +40 \%\end{aligned}$, independently from the stop mass, the stop-chargino mass splitting and the $\lambda_{33 i}^{\prime \prime}$ value.

\section{Systematic uncertainty from PDF}

Systematic uncertainties due to PDFs are evaluated by computing the cross sections of the two processes $2 b 2 j$ and $6 b 2 j$ at the center of mass energy of $14 \mathrm{TeV}$ with MADGRAPH5_AMC@NLO using two different PDF sets: NNPDF23LO1 [100], CTEQ6L [101]. The estimation of these uncertainties is performed similarly to the evaluation of the scale uncertainty, for three values of the stop mass, $m_{\tilde{t}}=600,800$ and $1000 \mathrm{GeV}$, three stop-chargino mass splitting equal to 50, 100 and $150 \mathrm{GeV}$ and three RPV $\lambda_{33 i}^{\prime \prime}$ couplings corresponding to $10^{-1}, 10^{-3}$ and $10^{-6}$. The result appears to be slightly dependent on $m_{\tilde{t}}$. The resulting relative variation in cross sections is found to be around $24 \%$ for $m_{\tilde{t}}=600$ $\mathrm{GeV}, 28 \%$ for $m_{\tilde{t}}=800 \mathrm{GeV}$ and $32 \%$ for $m_{\tilde{t}}=1 \mathrm{TeV}$.

Finally, we note that both PDF and scale uncertainties associated with the $2 b 2 j$ final state process are consistent within $2 \%$ with the ones found for the $6 b 2 j$ final state: this result allows us to assume the same order of magnitude for the uncertainty associated with the other RPV-processes listed in Sec. III B. 


\section{B. Final states sensitivity to $\lambda_{33 i}^{\prime \prime}$}

As can be seen from Figs. 2 and 3, the various cross-sections vary over several orders of magnitude due to a very high sensitivity to $\lambda_{33 i}^{\prime \prime}$. The extreme values of $\lambda_{33 i}^{\prime \prime}$ feature a reversed hierarchy of the contributions of the different final states. The most striking aspect is that the busiest $2 t 4 b 2 j$ final state dominates for extremely small values $\mathcal{O}\left(10^{-7}-10^{-5}\right)$ of $\lambda_{33 i}^{\prime \prime}$ while the $2 b 2 j, 4 b 2 j$ and $6 b 2 j$ final states dominate for $\lambda_{33 i}^{\prime \prime}$ of $\mathcal{O}\left(10^{-3}-10^{-1}\right)$, yet with comparable cross-sections of order a few tens to a hundred femtobarns. Moreover, as shown on Figs. 4(a) and $5(\mathrm{a})$, the relative contributions of the dominant $2 b 2 j, 4 b 2 j$ and $6 b 2 j$ final states for large $\lambda_{33 i}^{\prime \prime}$ depend also on the stop chargino mass splitting, typically with a (reversed) hierarchy given by the $b$-quark multiplicity. The $4 b 2 j$ channel can be comparable to the two other channels but is rarely dominant. The $2 b 2 j$ will always eventually dominate for sufficiently large $\lambda_{33 i}^{\prime \prime} \gtrsim 10^{-2}$ (e.g. for $\lambda_{33 i}^{\prime \prime} \gtrsim 10^{-1}$ not shown on the figures, its dominance prevails for small to moderate ranges of mass splitting). In contrast, the $6 b 2 j$ channel dominates in a range of intermediate values of $\lambda_{33 i}^{\prime \prime} \gtrsim 10^{-3}$ when the mass splitting is moderate to large.

These features illustrate clearly the complementarity of the different final states in view of extracting information in the RPV-coupling/mass-splitting parameter space. The general trend of the sensitivity to $\lambda_{33 i}^{\prime \prime}$ can be understood qualitatively from the NWA expressions, Eqs. (4.18) through (4.23). From the asymptotic behavior of these NWA expressions at small $\lambda_{33 i}^{\prime \prime}$, in the regime $r_{1} \times\left(\lambda_{33 i}^{\prime \prime}\right)^{2} \ll 1$ and $r_{2} \times\left(\lambda_{33 i}^{\prime \prime}\right)^{2} \ll 1$, one sees that all the topless final state channels scale with $\left(\lambda_{33 i}^{\prime \prime}\right)^{4}$, the channels with one top scale with $\left(\lambda_{33 i}^{\prime \prime}\right)^{2}$ and the channel with two top-quarks tends to be constant in $\lambda_{33 i}^{\prime \prime}$, which explains the tremendous orders of magnitude difference in the cross-sections and the dominance of the RPC-likechannel. If the other extreme of asymptotically large $\lambda_{33 i}^{\prime \prime}$ were allowed, i.e. $\lambda_{33 i}^{\prime \prime} \gtrsim 1, r_{1} \times\left(\lambda_{33 i}^{\prime \prime}\right)^{2} \gg 1$ and $r_{2} \times\left(\lambda_{33 i}^{\prime \prime}\right)^{2} \gg 1$, then only the $262 j$ would survive, becoming almost $\lambda_{33 i}^{\prime \prime}$-independent, the other channels scaling with increasing inverse powers of $\lambda_{33 i}^{\prime \prime}$ for increasing multiplicity of $b$ - and $t$-quarks in the final state. In fact, if one remains in the domain of moderate values of $\lambda_{33 i}^{\prime \prime}$ the behavior becomes more sensitive to $r_{1}, r_{2}$. In our scenario $r_{2}$ is always very large, typically several orders of magnitude larger than $r_{1}$, due to the smallness of the decay width of $\Gamma\left(\chi^{+} \rightarrow \chi^{0} f_{2}^{\prime} \bar{f}_{2}\right)$ and to the fact that $\chi^{+}$and $\chi^{0}$ are almost degenerate. For the considered range of $\lambda_{33 i}^{\prime \prime}$ we are always in the regime $r_{2} \times\left(\lambda_{33 i}^{\prime \prime}\right)^{2} \gg 1$. Similarly, $r_{1}$ can become equally large but only in corners of the parameter space where the stop is 
almost degenerate with the chargino as seen from Eq. (4.13). This allows to understand the relative magnitudes of the various cross-sections shown on the figures. For instance the ratio $\sigma(6 b 2 j) / \sigma(2 b 2 j)$ scales with $r_{1}^{-2}\left(\lambda_{33 i}^{\prime \prime}\right)^{-4}$ and is indeed (much) larger than 1 even at the upper edge of the domain of Eq. (3.1), except when $r_{1}$ becomes large due to small stop-chargino mass splitting, eventually reversing the hierarchy between the two cross-sections consistently with the numerical behavior shown on Figs. 4(a) and 5(a). One can understand similarly the behavior of $\sigma(4 b 2 j)$ that is bounded essentially between the $2 b 2 j$ and $6 b 2 j$ cross-sections irrespective of the mass splitting. Note however that $\sigma(4 b 2 j) / \sigma(6 b 2 j)$ scales with $2 r_{1}\left(\lambda_{33 i}^{\prime \prime}\right)^{2}$, so that the $4 b 2 j$ channel can come to dominate over all the other channels for moderate mass splitting and a $\lambda_{33 i}^{\prime \prime}$ somewhat larger than the range we consider for the analysis. Turning to the final states containing one or two top-quarks, their tiny contribution in the upper part of the $\lambda_{33 i}^{\prime \prime}$ range, cf. Figs. 2 and 3 , is due to the size of $r_{2}$. For instance $\sigma(2 b 2 j) / \sigma(2 t 4 b 2 j)$ scales with $r_{1}^{2} r_{2}^{2}\left(\lambda_{33 i}^{\prime \prime}\right)^{8}$, but the large suppression for $\lambda_{33 i}^{\prime \prime} \lesssim 0.1$ is compensated for by a very large value of $r_{2} \approx \mathcal{O}\left(10^{7}\right)$ as a consequence of the compressed light chargino/neutralino sector.

We turn now to a quantitative discussion of the comparison between the full matrix element calculation and the NWA. Given the huge difference in the scaling of the various cross-sections and the variations over several orders of magnitudes, this comparison is an important cross-check of the results. We indeed find that the NWA works reasonably well in configurations where it is expected to do so [70-73]. We check first the scaling relations t-SR and b-SR given in Eqs. $(4.25,4.26)$, as these provide global tests that do not require the knowledge of the stop production cross-section nor the $r_{1}, r_{2}$ ratios. A systematic test of t-SR and b-SR using all the cross-sections in Table IV and in Table $\mathrm{V}$ gave a relative deviation of $10 \%$ or more from these scaling relations only in $\lesssim 9 \%$ of the cases, while a deviation of $\lesssim 5 \%$ obtains in $\sim 80 \%$ of the cases and a deviation of $\lesssim 1 \%$ in $\sim 66 \%$ of the cases. It is also instructive to identify the configurations where the NWA fails badly. We find that deviations of more than $30 \%$, reaching up to $135 \%$, occur in less than $5 \%$ of the cases and only for t-SR that involves long chain decays. These correspond to points of benchmark 1 having large values of $\lambda_{33 i}^{\prime \prime}$ and very small stop-chargino mass splitting, such as for $\lambda_{33 i}^{\prime \prime}=10^{-1}$ and $m_{\tilde{t}}-m_{\chi^{+}}=59$ and $11 \mathrm{GeV}$ and for $\lambda_{33 i}^{\prime \prime}=10^{-2}$ and $m_{\tilde{t}}-m_{\chi^{+}}=5 \mathrm{GeV}$, shown in Table IV. Such large deviations are in accord with the general expectations [70]. We have also checked the NWA for individual cross-sections. This allowed to disentangle the 
reasons for the differences from the results of the full matrix element calculations. Very good quantitative agreement is observed for the shortest decay chains, and for small values of $\lambda_{33 i}^{\prime \prime}$ and/or large mass splitting for longer decay chains. The cross-sections given by Eqs. (4.18 $-4.23)$ reproduce globally the behavior shown in Figs. 2 and 3.

We discuss now three spectrum configurations that are outside one or the other of the assumptions given in Eqs. $(2.11-2.13)$. The values of $m_{\tilde{t}}-m_{\chi^{+}}=-36$ or $-43 \mathrm{GeV}$, shown in Table IV, correspond to points violating Eq. (2.11) with an MSSM-LSP stop. As expected, in this case the $2 b 2 j$ channel largely dominates independently of the magnitude of $\lambda_{33 i}^{\prime \prime}$, the next-to-leading channel, $4 b 2 j$, being two to three orders of magnitude smaller. It is however noteworthy that the $2 b 2 j$ channel can be dominant even when Eq. (2.11) is satisfied, provided that the positive mass splitting $m_{\tilde{t}}-m_{\chi^{+}}$remains sufficiently small and $\lambda_{33 i}^{\prime \prime}$ sufficiently large. One sees this tendency from the $m_{\tilde{t}}-m_{\chi^{+}}=5$ and $11 \mathrm{GeV}$ points in Table IV for benchmark 1 . For instance, the $2 b 2 j$ channel can still be an order of magnitude greater than the total of the remaining channels for a stop/chargino mass splitting in excess of $10 \mathrm{GeV}$, as illustrated for $m_{\tilde{t}}-m_{\chi^{+}}=11 \mathrm{GeV}$ and $\lambda_{33 i}^{\prime \prime}=10^{-1}$. A smaller mass splitting, at the edge of the validity of Eq. (2.13), leads to even larger effects, as one can see in Table $\mathrm{V}$ by looking at the point $m_{\tilde{t}}-m_{\chi^{+}}=5 \mathrm{GeV}$ and $\lambda_{33 i}^{\prime \prime}=10^{-1}$. In this case the $2 b 2 j$ channel dominates the other channels by almost two orders of magnitude. A larger mass splitting would require larger values of $\lambda_{33 i}^{\prime \prime}$ to ensure the dominance of the $2 b 2 j$ channel. In fact there is a correlation between the mass splitting and the size of the RPV coupling that can be understood in terms of the NWA cross-section of Eq. (4.18): the $2 b 2 j$ channel becomes dominant, with a branching ratio close to one, when $r_{1} \times\left(\lambda_{33 i}^{\prime \prime}\right)^{2} \gg 1$, say $\mathcal{O}(10)$ or larger. Indeed, the ratio $r_{1}$ becomes large for small stop/chargino mass splitting due to phase-space suppression of the width $\Gamma\left(\tilde{t} \rightarrow \chi^{+} b\right)$, see Eq. (4.13), implying that $2 b 2 j$ can dominate for moderately small $\lambda_{33 i}^{\prime \prime}$. More generally the regime where $2 b 2 j$ dominates is characterized roughly by $\left|\lambda_{33 i}^{\prime \prime}\right| \gtrsim 3 \times r_{1}^{-1 / 2}$. The present LHC limits [31, 32, 41] where the $2 b 2 j$ dominance is assumed, can thus be interpreted as excluding either scenarios where the stop is the MSSM-LSP, or the domain delineated by the above relation in scenarios where a chargino and a neutralino are lighter than the stop.

If Eq. (2.13) is not satisfied but the mass splitting still larger than the $s$-quark or $d$-quark masses then the $\boldsymbol{\chi}$-RPV and RPC-like decays occur dominantly through the LFV channel $\tilde{t} \rightarrow s(d) \chi^{+}$(recall that we assume MFV). The effect is thus noticeable for the small values 
of $\lambda_{33 i}^{\prime \prime}$ where the $\boldsymbol{\chi}$-RPV or RPC-like decays are expected to dominate. This is illustrated for all values of $\lambda_{33 i}^{\prime \prime}$ with mass splitting of $1 \mathrm{GeV}$ in Table $\mathrm{V}$. There are two effects: for $\lambda_{33 i}^{\prime \prime}$ in the intermediate range $10^{-4}-10^{-2}$, the $2 b 2 j$ channel becomes largely dominant over the $6 b 2 j$ and $1 t 5 b 2 j$ channels contrary to the typical cases with larger stop/chargino mass splitting. In this intermediate $\lambda_{33 i}^{\prime \prime}$ range the LFV channels with smaller $b$-quark multiplicity and larger light jet multiplicity such as $4 b 4 j$ and $1 t 3 b 4 j$ final states have cross-sections comparable to that of the $2 b 2 j$ channel given the size of the corresponding CKM mixing angles. In contrast, in the range $10^{-7} \lesssim \lambda_{33 i}^{\prime \prime} \lesssim 10^{-5}$ the cross-sections for all the final states listed in Table I become suppressed as can be seen in the corresponding blocks of Table $\mathrm{V}$ and mass splitting of $1 \mathrm{GeV}$ indicating that the dominant channel corresponds now to the LFV RPC-like-RPC-like final state $2 t 2 b 4 j$. The study of final states with more light quarks and less $b$-quark multiplicity can thus be motivated in the context of an inclusive search comprising the very narrow part of the parameter space having an extremely compressed $\tilde{t} / \chi^{+}$spectrum.

Last but not least, we consider the case where Eq. (2.12) is not satisfied. The decay channel $\tilde{t} \rightarrow t \chi^{0}\left(\chi_{2}^{0}\right)$ is now open leading to $4 t 2 b 2 j$ final states. A detailed study of this channel is outside the scope of the present paper and we do not give here the corresponding cross-section. Its is however interesting to note the indirect effect of this channel on the cross-sections given in Tables IV and V. Indeed, the expected drop of the latter when the top-neutralino channel sets in is found to remain relatively moderate. For instance, comparing the points $m_{\tilde{t}}-m_{\chi^{+}}=146 \mathrm{GeV}$ and $194 \mathrm{GeV}$ of Table IV one sees that the drop in the leading cross-sections $6 b 2 j, 1 t 5 b 2 j$ and $2 t 4 b 2 j$ is by a factor of order $2-2.5$ or less, depending on the magnitude of $\lambda_{33 i}^{\prime \prime}$. Similar effects are found for benchmark 2 , as seen from a comparison of the points $m_{\tilde{t}}-m_{\chi^{+}}=143 \mathrm{GeV}$ and $239 \mathrm{GeV}$ of Table V. This suggests that the final states considered in the present study can still contribute to signatures outside the specific mass configurations that we relied on.

To conclude this section, we stress the main point of the analysis: if part of the chargino/neutralino sector is lighter than the lightest stop, channels with different jet multiplicities probe dominantly different ranges of the RPV coupling. This is due to a distinct dependence on $\lambda_{33 i}^{\prime \prime}$ of the various decay widths and branching ratios, thus triggering the dominance of different channels for different values of this coupling. We depict this general feature schematically in Fig. 6 for a typical configuration, keeping in mind that the actual 
dominance ranges can change depending on the masses and RPC couplings. The analytical expressions for the cross-sections in terms of the RPV coupling and decay widths in the RPC sector given in Sec. IV allow a clear qualitative understanding of these features. The

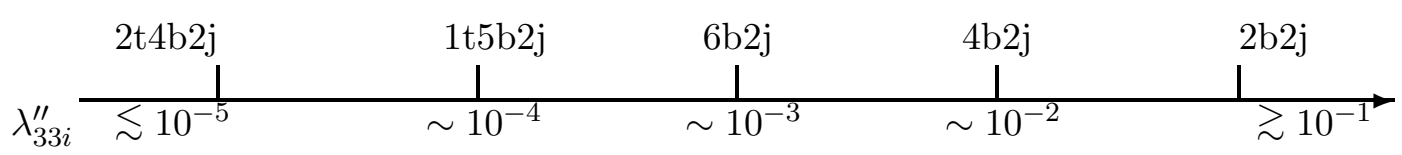

FIG. 6: Schematic illustration of $\lambda_{33 i}^{\prime \prime}$ as a signature generator; different magnitudes of this coupling favor different final states.

RPV coupling thus plays the role of a signature generator.

\section{RPV FINAL STATES AND SM BACKGROUND: A DISCUSSION}

The LHC is currently in its Run 2 data taking period, which started in 2015 and it is now providing proton-proton collisions at $\sqrt{s}=13 \mathrm{TeV}$ to both ATLAS and CMS, continuously improving in the delivered peak luminosity. With the current schedule, $100 \mathrm{fb}^{-1}$ of data and a possible further push in the center-of-mass energy to $\sqrt{s}=14 \mathrm{TeV}$, both ATLAS and CMS will be able to carry the BSM searches which are the core of the LHC Run 2 physics program. In what follows we give a brief overview of how the different RPV signatures, which are the focus of this paper, are, or can be looked for at the LHC. For the different final states treated, $2 b 2 j, 4 b 2 j, 6 b 2 j, 1 t 5 b 2 j$ and $2 t 4 b 2 j$, we either review the current experimental analyses, or, for those channels where no experimental analyses have been performed yet, we propose, based on similar existing analyses, a search strategy with a list of SM backgrounds which could impact their sensitivities.

\section{A. $2 b 2 j$}

Direct production of stop quarks with a subsequent RPV decay into two jets has been searched for at LEP and Tevatron, where a 95\% upper limit on the mass of such particles was set to respectively $82.5 \mathrm{GeV}$ [102] and $100 \mathrm{GeV}$ [103]. As pointed out by Ref. [104] first searches for stop production at the LHC did not succeed on being sensitive to any stop mass until the trigger strategy changed from using high transverse momentum $\left(p_{T}\right)$ multi-jet 
triggers, which had the effect of shaping the background towards high masses, to triggering on the totality of the hadronic energy deposited in the calorimeter (ATLAS [105]), a variable less correlated to the two masses of the di-jet resonances, or lower $p_{T}$ jets (CMS [41]). Both ATLAS and CMS have looked for stop production pairs final state where the two stops decay into $\bar{b} \bar{s}+b s$. The two stop-quark resonances are identified as wide hadronic calorimeter "fat" jets with a cone size, $R$, of the order of 1,1.5 [106]. Given that the characteristic distance between the two particles stemmed from a resonance, $\Delta R$, is of the order $2 m / p_{T}$, where $m$ is the stop-quark mass and $p_{T}$ its transverse momentum, this kind of signature allows to access a relatively low mass spectra, where most of the center of mass energy goes to the boost of the produced resonance pair.

The main challenge for hadronic jet based searches is to understand the normalizations and shapes of the multijet background, this has been shown to be possible using data-driven techniques [107]. The discrimination between signal and background is done by exploiting kinematic quantities such as the value of the reconstructed fat jet masses, which is the same for the two fat jets from stop pair production, and other jet substructure properties such as the difference in $p_{T}$ between the two subjets identified by un-doing the last step of the fat jet clustering, more pronounced in multijet events (see Ref. [32] and reference therein). Also fundamental to reduce multi-jet background, b-tagging algorithms are used to identify the presence of jets issued from the hadronization of $b$-quarks [108, 109]. After bump hunting, the two LHC experiments could exclude at 95\% Confidence Level stop quark production for masses up to $345 \mathrm{GeV}$ (ATLAS) [32] and $385 \mathrm{GeV}(\mathrm{CMS})$ [41]. This final state is sensitive not only to the value of the stop mass in the case the stop is the LSP, but if the lightest neutralino and the lightest chargino are lighter than the stop quark, then this class of analyses could be sensitive to the hardest part of the $\lambda_{332}^{\prime \prime}$ spectrum considered, as shown in Fig. 2, see also Section VIB.

\section{B. $4 b 2 j$ and $6 b 2 j$}

As discussed in section VIB the topless multi- $b$-jet signatures saturate the stop quark branching ratio for intermediate values of $\lambda_{33 i}^{\prime \prime} \gtrsim 10^{-3}$. The $4 b 2 j$ and $6 b 2 j$ signatures have the highest cross sections at large $\lambda_{33 i}^{\prime \prime}$ value when the differences between the stop and the chargino/neutralino masses are maximal, see Figs 2 and 4. In this scenario, for low stop 
masses, such that $2 m / p_{T}$ is $O(1)$, the same strategy as the searches in the $2 b 2 j$ final state can be used, where two structured large sized hadronic jets of particles are produced back to back. This facilitates the task of eliminating the combinatorial background that arises when the presence of multiple reconstructed objects in the final state does not allow to assign them to one of the particle originating the decay. This results in a poor reconstruction of the resonant peaks. Moreover the presence of resonances within the fat jets helps discriminating against the background when using the value of the reconstructed invariant mass of the stop and chargino candidates and of more specific jet substructure related quantities such as the $k_{t}$ splitting scale and $n$-subjettiness (see Ref. [110] and references therein). It has also been recently suggested [111] that jet reconstruction techniques based on a mass-jump clustering algorithm with variable size can be used to reconstruct multi-jet resonance in very busy environment as the one produced by boosted stop squarks decay into $4 b 2 j$ or $6 b 2 j$ final states.

In the case of resolved regime, where most jets from the stop and chargino decay are reconstructed, the signal is characterized by events with high jet and $b$-jet multiplicity. If no $b$-tagging is required at the analysis level, the signal, even for stop masses of about one $\mathrm{TeV}$, although it would present very high jet multiplicity, would be still swamped by the presence of a large multi-jet background [112].

When the $b$-jet identification is used, the physics processes that could mimic RPV stop signal, include any resonant multi- $b$-jet production such as $t \bar{t}+X$, abundantly produced in the $\sqrt{s}=14 \mathrm{TeV}$ proton-proton collisions at the LHC. Background processes to this final state include $t \bar{t}$ plus light and heavy flavored jets, $t \bar{t}$ plus vector boson, and $t \bar{t} H(\rightarrow b \bar{b})$ production, where both top quarks decay fully hadronically. The inclusive $t \bar{t}$ cross section is known at NNLO in QCD including resummation of soft gluon terms at next-to-leadinglogarithmic (NNLL) [113]; at $\sqrt{s}=14 \mathrm{TeV}, \sigma_{t \bar{t}}=954_{-34}^{+23}\left(\right.$ scale) ${ }_{-18}^{+16}($ pdf $) \mathrm{pb}, 45.7 \%$ of which decays fully hadronically [11]. At the analysis level when asking for more than $2 b$-tagged jets it is more likely to select events from processes where extra heavy flavors are produced. For $\sqrt{s}=14 \mathrm{TeV}, \sigma_{t \bar{t}+b \bar{b}}$ is known at NLO to be $2.63_{-70}^{+86}$ (scale) pb [114], $\sigma_{t \bar{t} \rightarrow Z}$ is also known at NLO with a value of $1057_{-104}^{+110}(\text { scale })_{-25}^{+20}(\mathrm{pdf}) \mathrm{fb}$ [115] while $\sigma_{t \bar{t} \rightarrow W}=769_{-170}^{+228}(\mathrm{scale})_{-61}^{+54}(\mathrm{pdf})$ $\mathrm{fb}$ [116]. As for the associated top and Higgs production, $t \bar{t} H$ cross section is known at NNLO in QCD and EW plus resummation of soft gluon at NNLL; at $\sqrt{s}=14 \mathrm{TeV}, \sigma_{t \bar{t} H(H \rightarrow b \bar{b})}=$ $625_{-42}^{+29}(\text { scale })_{-14}^{+14}(\mathrm{pdf}) \mathrm{fb}[117]$. Recent LHC analyses at $\sqrt{s}=8 \mathrm{TeV}$ [107] show how by 
just selecting a high multi-jet $(\approx 8)$ and $b$-jet multiplicity $(\approx 4)$ the background composition is made at about $80 \%$ of multijets. This background has little resemblance with multiresonant production and can be discriminated using multi-variate analysis which exploits different energy regime, event shape, using quantities such as centrality, aplanarity and the mass of the reconstructed top quark candidates. Such kind of analysis needs to control the uncertainties on the main top-like background and at the same time removing as much as possible multi-jet background.

Despite the large background from multi-jet events, given the large energy deposit in the hadronic calorimeter from the decay products of the pair of massive resonances, and the large presence of jets coming from $b$-quark, as also stated in Ref. [43], this channel is very promising. Searches at hadron colliders for gluino pair production and subsequent RPV decay into $t b s$, where similar final states are investigated, could already show sensitivity to this channel [38].

\section{C. $1 t 5 b 2 j$ and $2 t 4 b 2 j$}

Signatures with decays into top quarks saturate the branching ratio for $\lambda_{33 i}^{\prime \prime}<10^{-5}$, as already discussed in Section VIB. These signatures are interesting since the presence of a lepton from the top quark decay can be easily identified at trigger level and used to eliminate the otherwise overwhelming multi-jet background, such as in the fully hadronic signatures. After selecting at least six $b$-tagged jets in addition to at least two light-jets and one lepton, for the case of $1 t 5 b 2 j$, or two leptons, for the case of $2 t 4 b 2 j$, the main irreducible background arises from $t \bar{t}+$ jets and $t \bar{t} H(\rightarrow b \bar{b})+$ jets. For simplicity in this discussion we limit ourself to analyzing the dileptonic top quark decay for the $2 t 4 b 2 j$ final state to allow discussing the backgrounds composition to both final states. In this case, the main background for both final states comes from $t \bar{t}+b \bar{b} b \bar{b}+$ jets. The LO cross section for $t \bar{t}+b \bar{b} b \bar{b}$ at $\sqrt{s}=14 \mathrm{TeV}$, estimated with MADGrAPH5_AMC@NLO using LHAPDF 6.1 .6 [118], is $290_{-160}^{+400}(\text { scale })_{-50}^{+90}$ (pdf) fb, comparable with the signal cross section in the low $\lambda_{33 i}^{\prime \prime}$ regime, see for example $\lambda_{33 i}^{\prime \prime}<10^{-5}$ in Table IV. The presence of neutrinos and the large jet multiplicity present in this final state makes it difficult to reconstruct completely the final state, i.e. to assign unambiguously reconstructed leptons and jets to the stop and anti-stop decays. This effect weakens the power of distributions such as the invariant mass of the reconstructed stop 
and chargino candidates, to discriminate signal and background events. The large energy deposited in the detectors, equal approximately to twice the stop mass, could have the role of the missing transverse envergy for RPC searches, to discriminate signal against the softer top quark pair production, using for example the transverse energy of the event $\left(H_{T}\right)$. On the other hand the softer part of the $H_{T}$ distribution can be useful to control the effect of major systematic uncertainties, especially the large theoretical uncertainties on $t \bar{t}+b \bar{b} b \bar{b}$ cross section, on the LHC sensitivity for this channel. This class of final states can use the analysis techniques developed for $t t H(H \rightarrow b \bar{b})$ searches and that need to be extended to higher jet multiplicity.

As for the case of $4 b 2 j$ and $6 b 2 j$, both ATLAS and CMS searches for gluino pair production and subsequent RPV decay to a pair of top quarks and jets through the R-parity violating decay of either the neutralino into three quarks or the top squark into SM quarks [30, 38], could already be reinterpreted as limits on $\lambda_{33 i}^{\prime \prime}$ using this channel.

\section{CONCLUSION \& OUTLOOK}

The ever stronger exclusion limits on SUSY particle masses from negative searches at the LHC seem to disfavor, if not to rule out, low energy supersymmetry as the correct theory beyond the SM. However, one should not lose sight of the distinction between SUSY as a general framework and its various possible model realizations. Only a class of the latter, leading to RPC signatures with striking missing energy, is being heavily excluded by the LHC. If RPV baryon number violating couplings are allowed, a class of signatures, generally with high jet and lepton multiplicity and no missing energy is expected at the LHC. Searches for such RPV signals usually assume that the shortest chain particle decays involving RPV vertices have $100 \% B R$. Previous works have already pointed out, for a particular case involving decaying stops with $\lambda_{331}^{\prime \prime}, \lambda_{332}^{\prime \prime} \neq 0$, the existence of a region in the mass parameter space for which longer decay chains and richer final states than the plain $\tilde{t} \rightarrow b s, b d$ can originate.

In this paper we described how different stop-pair final states arise when different values of the RPV coupling and different supersymmetric particle mass splittings are considered. This is exhaustively investigated for the case of proton-proton collisions at center-of-mass

energy of $\sqrt{s}=14 \mathrm{TeV}$. After having defined a set of working assumptions concerning the 
mass hierarchy and the allowed range of $\lambda_{33 i}^{\prime \prime}$, we examined the sensitivity of the stop decay branching ratios to $\lambda_{33 i}^{\prime \prime}$, first analytically by means of the NWA approximation showing that the variation of $\lambda_{33 i}^{\prime \prime}$ over several orders of magnitude triggers the dominance of very different final states, then numerically relying on automated matrix element calculations.

Using for the latter a bottom-bottom approach in the phenomenological MSSM, we generated the full mass spectrum and couplings and identified two benchmark points taking into account all possible constraints ranging from the measured Higgs mass to the experimental low energy constraints. For these two benchmark points we estimated the cross sections for the relevant final states differing by the number of heavy and light flavored quarks $(2 b 2 j$, $4 b 2 j, 6 b 2 j, 1 t 5 b 2 j$ and $2 t 4 b 2 j$ ), as a function of $\lambda_{33 i}^{\prime \prime}$ and the stop/chargino mass splitting, confirming numerically what is seen analytically with the NWA approximation. Finally we discussed the phenomenology of the RPV stop production and decays and its rich experimental signatures, stressing that the smaller the values of $\lambda_{33 i}^{\prime \prime}$ the larger the quark mutliplicity

of the dominant final states. Some of these final states having so far not been extensively looked at experimentally, we briefly discussed how they can be searched for at the LHC.

While other studies, including single stop resonant and associate productions as well as the increaslingly strict limits on displaced vertices and long-lived particles, contribute to narrowing down the viable RPV scenarios, significant parts of the parameter space remain to be explored. As such, an exciting possibility still lies ahead, that a light part of the MSSM spectrum, a key issue for the naturalness of SUSY, may be stashed in the present and future LHC data.

\section{Acknowledgments}

We thank Florian Staub for very useful discussions and substantial help in the use of SARAH and implementation of the SPHENo package for the RPV-MSSM. We also would like to thank Stephane Lavignac for helpful discussions, as well as Jared Evans and Angelo Monteux for the various comments that helped improve the present paper.

This work has been carried out thanks to the support of the OCEVU Labex (ANR-11LABX-0060) and the A MIDEX project (ANR-11-IDEX-0001-02) funded by the "Investisse- 
ments d'Avenir" French government program managed by the ANR.

[1] F. Englert and R. Brout, "Broken Symmetry and the Mass of Gauge Vector Mesons," Phys. Rev. Lett. 13 (1964) 321.

[2] P. W. Higgs, "Broken Symmetries and the Masses of Gauge Bosons," Phys. Rev. Lett. 13 (1964) 508.

[3] P. W. Higgs, "Broken Symmetries, Massless Particles and Gauge Fields," Phys. Lett. 12 (1964) 132.

[4] G. S. Guralnik, C. R. Hagen, and T. B. W. Kibble, "Global Conservation Laws and Mass-less Particles," Phys. Rev. Lett. 13 (1964) 585.

[5] ATLAS Collaboration, "Observation of a new particle in the search for the Standard Model Higgs boson with the ATLAS detector at the LHC," Phys. Lett. B 716 (2012) 1-29, arXiv:1207.7214 [hep-ex].

[6] CMS Collaboration, C. Collaboration, "Observation of a new boson at a mass of $125 \mathrm{GeV}$ with the CMS experiment at the LHC," Phys. Lett. B 716 (2012) 30-61, arXiv:1207.7235 [hep-ex].

[7] ATLAS, CMS Collaboration, "Combined Measurement of the Higgs Boson Mass in $p p$ Collisions at $\sqrt{s}=7$ and $8 \mathrm{TeV}$ with the ATLAS and CMS Experiments," Phys. Rev. Lett. 114 (2015) 191803, arXiv:1503.07589 [hep-ex].

[8] LHCb Collaboration, "Test of lepton universality using $B^{+} \rightarrow K^{+} \ell^{+} \ell^{-}$decays," Phys. Rev. Lett. 113 (2014) 151601, arXiv:1406.6482 [hep-ex].

[9] LHCb Collaboration, "Angular analysis of the $B^{0} \rightarrow K^{* 0} \mu^{+} \mu^{-}$decay using $3 \mathrm{fb}^{-1}$ of integrated luminosity," JHEP 02 (2016) 104, arXiv:1512.04442 [hep-ex].

[10] S. Descotes-Genon, L. Hofer, J. Matias, and J. Virto, "Global analysis of $b \rightarrow$ sll anomalies," JHEP 06 (2016) 092, arXiv:1510.04239 [hep-ph].

[11] Particle Data Group Collaboration, K. A. Olive et al., "Review of Particle Physics," Chin. Phys. C 38 (2014) 090001.

[12] F. F. Deppisch, P. S. Bhupal Dev, and A. Pilaftsis, "Neutrinos and Collider Physics," New J. Phys. 17 no. 7, (2015) 075019, arXiv:1502.06541 [hep-ph].

[13] Yu. A. Golfand and E. P. Likhtman, "Extension of the Algebra of Poincare Group 
Generators and Violation of p Invariance," JETP Lett. 13 (1971) 323-326. [Pisma Zh. Eksp. Teor. Fiz.13,452(1971)].

[14] P. Ramond, "Dual Theory for Free Fermions," Phys. Rev. D 3 (1971) 2415-2418.

[15] A. Neveu and J. H. Schwarz, "Quark Model of Dual Pions," Phys. Rev. D 4 (1971) 1109-1111.

[16] J.-L. Gervais and B. Sakita, "Functional integral approach to dual resonance theory," Phys. Rev. D 4 (1971) 2291-2308.

[17] D. V. Volkov and V. P. Akulov, "Is the Neutrino a Goldstone Particle?," Phys. Lett. B 46 (1973) 109-110.

[18] J. Wess and B. Zumino, "A Lagrangian Model Invariant Under Supergauge Transformations," Phys. Lett. B 49 (1974) 52.

[19] P. Fayet, "Supergauge Invariant Extension of the Higgs Mechanism and a Model for the electron and Its Neutrino," Nucl. Phys. B 90 (1975) 104-124.

[20] A. Salam and J. A. Strathdee, "Supersymmetry and Fermion Number Conservation," Nucl. Phys. B 87 (1975) 85-92.

[21] J. A. Evans and Y. Kats, "LHC Coverage of RPV MSSM with Light Stops," JHEP 04 (2013) 028, arXiv:1209.0764 [hep-ph].

[22] C. Csaki, E. Kuflik, O. Slone, and T. Volansky, "Models of Dynamical R-Parity Violation," JHEP 06 (2015) 045, arXiv:1502.03096 [hep-ph].

[23] R. N. Mohapatra, "Supersymmetry and R-parity: an Overview," Phys. Scripta 90 (2015) 088004, arXiv:1503.06478 [hep-ph].

[24] A. E. Nelson and N. Seiberg, "R symmetry breaking versus supersymmetry breaking," Nucl. Phys. B 416 (1994) 46-62, arXiv:hep-ph/9309299 [hep-ph].

[25] L. E. Ibanez and G. G. Ross, "Discrete gauge symmetries and the origin of baryon and lepton number conservation in supersymmetric versions of the standard model," Nucl. Phys. B 368 (1992) 3-37.

[26] ATLAS Collaboration, "Search for supersymmetry with two same-sign leptons or three leptons using $13.2 \mathrm{fb}^{1}$ of $\sqrt{s}=13 \mathrm{TeV} p p$ collision data collected by the ATLAS detector," Aug, 2016. http://cds.cern.ch/record/2205745. ATLAS-CONF-2016-037.

[27] ATLAS Collaboration, "Search for a heavy narrow resonance decaying to $e \mu, e \tau$, or $\mu \tau$ with the ATLAS detector in $\sqrt{s}=7 \mathrm{TeV} p p$ collisions at the LHC," 
Phys. Lett. B 723 (2013) 15-32, arXiv:1212.1272.

[28] ATLAS Collaboration, "Search for supersymmetry in events with four or more leptons in $\sqrt{s}=13 \mathrm{TeV}$ pp collisions using $13.3 \mathrm{fb}^{1}$ of ATLAS data," Aug, 2016.

http://cds.cern. ch/record/2206245. ATLAS-CONF-2016-075.

[29] ATLAS Collaboration, "Search for massive supersymmetric particles in multi-jet final states produced in pp collisions at $\sqrt{s}=13 \mathrm{TeV}$ using the ATLAS detector at the LHC," Aug, 2016. http://cds.cern.ch/record/2206149. ATLAS-CONF-2016-057.

[30] ATLAS Collaboration, "Search for new physics in a lepton plus high jet multiplicity final state with the ATLAS experiment using $\sqrt{s}=13 \mathrm{TeV}$ proton-proton collision data," Aug, 2016. https://cds.cern. ch/record/2211457.

[31] ATLAS Collaboration, "A search for top squarks with R-parity-violating decays to all-hadronic final states with the ATLAS detector in $\sqrt{s}=8 \mathrm{TeV}$ proton-proton collisions," arXiv: 1601.07453 [hep-ex].

[32] ATLAS Collaboration, "A search for R-parity violating decays of the top squark in four jet final states with the ATLAS detector at $\sqrt{s}=13 \mathrm{TeV}, "$ May, 2016. http://cds . cern. ch/record/2152392. ATLAS-CONF-2016-022.

[33] CMS Collaboration, "Search for top squarks in $R$-parity-violating supersymmetry using three or more leptons and b-tagged jets," Phys. Rev. Lett. 111 no. 22, (2013) 221801, arXiv:1306.6643 [hep-ex].

[34] CMS Collaboration, "Search for new physics in events with same-sign dileptons and jets in pp collisions at $\sqrt{s}=8 \mathrm{TeV}$," JHEP 01 (2014) 163, arXiv:1311.6736. [Erratum: JHEP01,014(2015)].

[35] CMS Collaboration, "Search for RPV supersymmetry with three or more leptons and b-tags,". CMS-PAS-SUS-12-027.

[36] CMS Collaboration, "Searches for light- and heavy-flavour three-jet resonances in pp collisions at $\sqrt{s}=8 \mathrm{TeV}, "$ Phys. Lett. B 730 (2014) 193-214, arXiv:1311.1799 [hep-ex].

[37] CMS Collaboration, "Search for RPV SUSY in the four-lepton final state,". CMS-PAS-SUS-13-010.

[38] CMS Collaboration, "Searches for R-parity-violating supersymmetry in pp collisions at $\sqrt{s}=8 \mathrm{TeV}$ in final states with 0-4 leptons," Submitted to: Phys. Rev. D (2016), arXiv:1606.08076 [hep-ex]. 
[39] CMS Collaboration, "Search for R-parity-violating SUSY in final states with zero or one lepton and large multiplicity of jets and b-tagged jets," 2016. https://cds . cern. ch/record/2205147. CMS-PAS-SUS-16-013.

[40] CMS Collaboration, "Search for R-parity violating supersymmetry with displaced vertices," 2016. https://cds.cern.ch/record/2160356. CMS-PAS-SUS-14-020.

[41] CMS Collaboration, "Search for pair-produced resonances decaying to jet pairs in protonproton collisions at $\sqrt{s}=8 \mathrm{TeV}, "$ Phys. Lett. B 747 (2015) 98-119, arXiv:1412.7706 [hep-ex].

[42] R. Barbier et al., "R-parity violating supersymmetry," Phys. Rept. 420 (2005) 1-202, arXiv:hep-ph/0406039 [hep-ph].

[43] J. A. Evans, "A Swarm of Bs," JHEP 1408 (2014) 073, arXiv:1402.4481 [hep-ph].

[44] Z. Liu and B. Tweedie, "The Fate of Long-Lived Superparticles with Hadronic Decays after LHC Run 1," JHEP 06 (2015) 042, arXiv:1503.05923 [hep-ph].

[45] C. Csaki, E. Kuflik, S. Lombardo, O. Slone, and T. Volansky, "Phenomenology of a Long-Lived LSP with R-Parity Violation," JHEP 08 (2015) 016, arXiv:1505.00784 [hep-ph].

[46] A. Monteux, "New signatures and limits on R-parity violation from resonant squark production," JHEP 03 (2016) 216, arXiv:1601.03737 [hep-ph].

[47] G. D'Ambrosio, G. F. Giudice, G. Isidori, and A. Strumia, "Minimal flavor violation: An Effective field theory approach," Nucl. Phys. B645 (2002) 155-187, arXiv:hep-ph/0207036 [hep-ph].

[48] CMS Collaboration Collaboration, "Search for direct top squark pair production in the fully hadronic final state in proton-proton collisions at $\sqrt{s}=13 \mathrm{TeV}$ corresponding to an integrated luminosity of 12.9/fb," 2016. https://cds.cern.ch/record/2205176. CMS-PAS-SUS-16-029.

[49] CMS Collaboration, "Search for direct top squark pair production in the single lepton final state at $\sqrt{s}=13 \mathrm{TeV}, " 2016$. https://cds.cern.ch/record/2205271. CMS-PAS-SUS-16-028.

[50] ATLAS Collaboration, "Search for top squarks in final states with one isolated lepton, jets, and missing transverse momentum in $\sqrt{s}=13 \mathrm{TeV}$ pp collisions with the ATLAS detector," Aug, 2016. https://cds.cern.ch/record/2206132. ATLAS-CONF-2016-050. 
[51] ATLAS Collaboration, "Search for the Supersymmetric Partner of the Top Quark in the Jets+Emiss Final State at $\sqrt{s}=13 \mathrm{TeV}, "$ Aug, 2016. https://cds. cern. ch/record/2206250. ATLAS-CONF-2016-077.

[52] E. Nikolidakis and C. Smith, "Minimal Flavor Violation, Seesaw, and R-parity," Phys. Rev. D 77 (2008) 015021, arXiv:0710.3129 [hep-ph].

[53] C. Csaki, Y. Grossman, and B. Heidenreich, "MFV SUSY: A Natural Theory for R-Parity Violation," Phys. Rev. D 85 (2012) 095009, arXiv:1111.1239 [hep-ph].

[54] Y. Mambrini and G. Moultaka, "Infrared quasifixed point structure in extended Yukawa sectors and application to R parity violation," Phys. Rev. D 65 (2002) 115011, arXiv:hep-ph/0112353 [hep-ph].

[55] Y. Mambrini and G. Moultaka, "Comment on 'Infrared fixed point structure in minimal supersymmetric standard model with baryon and lepton number violation'," Phys. Rev. D 65 (2002) 058901, arXiv:hep-ph/0103270 [hep-ph].

[56] N. Chamoun, H. K. Dreiner, F. Staub, and T. Stefaniak, "Resurrecting light stops after the 125 GeV Higgs in the baryon number violating CMSSM," JHEP 08 (2014) 142, arXiv:1407.2248 [hep-ph].

[57] M. Papucci, J. T. Ruderman, and A. Weiler, "Natural SUSY Endures," JHEP 09 (2012) 035, arXiv:1110.6926 [hep-ph].

[58] S. Lola, P. Osland, and A. R. Raklev, "Radiative gravitino decays from R-parity violation," Phys. Lett. B 656 (2007) 83-90, arXiv:0707.2510 [hep-ph].

[59] F. Takayama and M. Yamaguchi, "Gravitino dark matter without R-parity," Phys. Lett. B 485 (2000) 388-392, arXiv:hep-ph/0005214 [hep-ph].

[60] G. Moreau and M. Chemtob, "R-parity violation and the cosmological gravitino problem," Phys. Rev. D 65 (2002) 024033, arXiv:hep-ph/0107286 [hep-ph].

[61] W. Beenakker, M. Kramer, T. Plehn, M. Spira, and P. Zerwas, "Stop production at hadron colliders," Nucl. Phys. B 515 (1998) 3-14, arXiv:hep-ph/9710451 [hep-ph].

[62] W. Beenakker, S. Brensing, M. Kramer, A. Kulesza, E. Laenen, and I. Niessen, "Supersymmetric top and bottom squark production at hadron colliders," JHEP 08 (2010) 098, arXiv:1006.4771 [hep-ph].

[63] W. Beenakker, S. Brensing, M. n. Kramer, A. Kulesza, E. Laenen, L. Motyka, and I. Niessen, "Squark and Gluino Hadroproduction," 
Int. J. Mod. Phys. A 26 (2011) 2637-2664, arXiv:1105.1110 [hep-ph].

[64] G. Durieux and C. Smith, "The same-sign top signature of R-parity violation," JHEP 10 (2013) 068, arXiv:1307.1355 [hep-ph].

[65] ATLAS Collaboration, G. Aad et al., "Search for new phenomena in final states with an energetic jet and large missing transverse momentum in pp collisions at $\sqrt{s}=8 \mathrm{TeV}$ with the ATLAS detector," Eur. Phys. J. C75 no. 7, (2015) 299, arXiv:1502.01518 [hep-ex]. [Erratum: Eur. Phys. J.C75,no.9,408(2015)].

[66] K. Choi, K. Hwang, and J. S. Lee, "Constraints on R-parity and B violating couplings in gauge mediated supersymmetry breaking models," Phys. Lett. B428 (1998) 129-135, arXiv:hep-ph/9802323 [hep-ph].

[67] ATLAS Collaboration, G. Aad et al., "Search for massive, long-lived particles using multitrack displaced vertices or displaced lepton pairs in pp collisions at $\sqrt{s}=8 \mathrm{TeV}$ with the ATLAS detector," Phys. Rev. D92 no. 7, (2015) 072004, arXiv: 1504.05162 [hep-ex].

[68] CMS Collaboration, V. Khachatryan et al., "Search for Long-Lived Neutral Particles Decaying to Quark-Antiquark Pairs in Proton-Proton Collisions at $\sqrt{s}=8 \mathrm{TeV}$," Phys. Rev. D91 no. 1, (2015) 012007, arXiv:1411.6530 [hep-ex].

[69] B. De Wit and J. Smith, Field Theory in Particle Physics Volume 1. North-Holland, Amsterdam, Netherlands, 1986.

[70] D. Berdine, N. Kauer, and D. Rainwater, "Breakdown of the Narrow Width Approximation for New Physics," Phys. Rev. Lett. 99 (2007) 111601, arXiv:hep-ph/0703058 [hep-ph].

[71] N. Kauer, "Narrow-width approximation limitations," Phys. Lett. B 649 (2007) 413-416, arXiv:hep-ph/0703077 [hep-ph].

[72] N. Kauer, "A Threshold-improved narrow-width approximation for BSM physics," JHEP 04 (2008) 055, arXiv:0708.1161 [hep-ph].

[73] C. F. Uhlemann and N. Kauer, "Narrow-width approximation accuracy," Nucl. Phys. B 814 (2009) 195-211, arXiv:0807.4112 [hep-ph].

[74] F. Staub, T. Ohl, W. Porod, and C. Speckner, "A Tool Box for Implementing Supersymmetric Models," Comput. Phys. Commun. 183 (2012) 2165-2206, arXiv:1109.5147 [hep-ph].

[75] W. Research, "Mathematica 11.0," 2010. 
[76] J. Alwall, R. Frederix, S. Frixione, V. Hirschi, F. Maltoni, O. Mattelaer, H. S. Shao, T. Stelzer, P. Torrielli, and M. Zaro, "The automated computation of tree-level and next-to-leading order differential cross sections, and their matching to parton shower simulations," JHEP 07 (2014) 079, arXiv:1405.0301 [hep-ph].

[77] W. Porod and F. Staub, "SPheno 3.1: Extensions including flavour, CP-phases and models beyond the MSSM," Comput.Phys.Commun. 183 (2012) 2458-2469, arXiv:1104.1573 [hep-ph].

[78] P. Bechtle, O. Brein, S. Heinemeyer, G. Weiglein, and K. E. Williams, "HiggsBounds: Confronting Arbitrary Higgs Sectors with Exclusion Bounds from LEP and the Tevatron," Comput. Phys. Commun. 181 (2010) 138-167, arXiv:0811.4169 [hep-ph].

[79] P. Bechtle, O. Brein, S. Heinemeyer, O. Stal, T. Stefaniak, G. Weiglein, and K. Williams, "Recent Developments in HiggsBounds and a Preview of HiggsSignals," PoS CHARGED2012 (2012) 024, arXiv:1301.2345 [hep-ph].

[80] P. Bechtle, S. Heinemeyer, O. Stl, T. Stefaniak, and G. Weiglein, "HiggsSignals: Confronting arbitrary Higgs sectors with measurements at the Tevatron and the LHC," Eur. Phys. J. C 74 no. 2, (2014) 2711, arXiv:1305.1933 [hep-ph].

[81] LHCb Collaboration, "First Evidence for the Decay $B_{s}^{0} \rightarrow \mu^{+} \mu^{-}$," Phys. Rev. Lett. 110 no. 2, (2013) 021801, arXiv:1211.2674 [hep-ex].

[82] LHCb Collaboration, "Measurement of the $B_{s}^{0} \rightarrow \mu^{+} \mu^{-}$branching fraction and search for $B^{0} \rightarrow \mu^{+} \mu^{-}$decays at the LHCb experiment," Phys. Rev. Lett. 111 (2013) 101805, arXiv:1307.5024 [hep-ex].

[83] CMS Collaboration, "Measurement of the $\mathrm{B}(\mathrm{s})$ to $\mathrm{mu}+\mathrm{mu}-$ branching fraction and search for B0 to mu+ mu- with the CMS Experiment," Phys. Rev. Lett. 111 (2013) 101804, arXiv:1307.5025 [hep-ex].

[84] H. K. Dreiner, K. Nickel, and F. Staub, "On the two-loop corrections to the Higgs mass in trilinear R-parity violation," Phys. Lett. B 742 (2015) 261-265, arXiv:1411.3731 [hep-ph].

[85] J. P. Miller, E. de Rafael, B. L. Roberts, and D. Stckinger, "Muon (g-2): Experiment and Theory," Ann. Rev. Nucl. Part. Sci. 62 (2012) 237-264.

[86] T. Ibrahim and P. Nath, "CP violation and the muon anomaly in N=1 supergravity," Phys. Rev. D61 (2000) 095008, arXiv:hep-ph/9907555 [hep-ph]. 
[87] D. Stockinger, "The Muon Magnetic Moment and Supersymmetry," J. Phys. G34 (2007) R45-R92, arXiv:hep-ph/0609168 [hep-ph].

[88] G.-C. Cho and K. Hagiwara, "Supersymmetry versus precision experiments revisited," Nucl. Phys. B 574 (2000) 623-674, arXiv:hep-ph/9912260 [hep-ph].

[89] S. Bertolini, F. Borzumati, A. Masiero, and G. Ridolfi, "Effects of supergravity induced electroweak breaking on rare B decays and mixings," Nucl. Phys. B 353 (1991) 591-649.

[90] M. Misiak et al., "Updated NNLO QCD predictions for the weak radiative $B$-meson decays," Phys. Rev. Lett. 114 no. 22, (2015) 221801, arXiv:1503.01789 [hep-ph].

[91] Heavy Flavor Averaging Group (HFAG) Collaboration, "Averages of $b$-hadron, $c$-hadron, and $\tau$-lepton properties as of summer 2014," arXiv:1412.7515 [hep-ex].

[92] H. K. Dreiner, K. Nickel, and F. Staub, " $B_{s, d}^{0}$ and $B X_{s}$ in the R-parity violating MSSM," Phys. Rev. D 88 no. 11, (2013) 115001, arXiv:1309.1735 [hep-ph].

[93] LHCb, CMS Collaboration, "Observation of the rare $B_{s}^{0} \rightarrow \mu^{+} \mu^{-}$decay from the combined analysis of CMS and LHCb data," Nature 522 (2015) 68-72, arXiv:1411.4413 [hep-ex].

[94] C. Bobeth, M. Gorbahn, T. Hermann, M. Misiak, E. Stamou, and M. Steinhauser, " $B_{s, d} \rightarrow l^{+} l^{-}$in the Standard Model with Reduced Theoretical Uncertainty," Phys. Rev. Lett. 112 (2014) 101801, arXiv:1311.0903 [hep-ph].

[95] C. Borschensky, M. Krmer, A. Kulesza, M. Mangano, S. Padhi, T. Plehn, and X. Portell, "Squark and gluino production cross sections in pp collisions at $\sqrt{s}=13,14,33$ and 100 TeV," Eur. Phys. J. C 74 no. 12, (2014) 3174, arXiv:1407.5066 [hep-ph].

[96] G. L. Kane and J. P. Leveille, "Experimental Constraints on Gluino Masses and Supersymmetric Theories," Phys. Lett. B 112 (1982) 227-232.

[97] P. R. Harrison and C. H. Llewellyn Smith, "Hadroproduction of Supersymmetric Particles," Nucl. Phys. B 213 (1983) 223. [Erratum: Nucl. Phys.B223,542(1983)].

[98] S. Dawson, E. Eichten, and C. Quigg, "Search for Supersymmetric Particles in Hadron Hadron Collisions," Phys. Rev. D 31 (1985) 1581.

[99] M. Bonvini, S. Marzani, J. Rojo, L. Rottoli, M. Ubiali, R. D. Ball, V. Bertone, S. Carrazza, and N. P. Hartland, "Parton distributions with threshold resummation," JHEP 09 (2015) 191, arXiv:1507.01006 [hep-ph].

[100] NNPDF Collaboration, "Progress in the NNPDF global analysis and the impact of the 
legacy HERA combination," PoS EPS-HEP2015 (2015) 506,

arXiv:1508.07731 [hep-ph].

[101] J. Pumplin, D. R. Stump, J. Huston, H. L. Lai, P. M. Nadolsky, and W. K. Tung, "New generation of parton distributions with uncertainties from global QCD analysis," JHEP 07 (2002) 012, arXiv:hep-ph/0201195 [hep-ph].

[102] ALEPH Collaboration, A. Heister et al., "Search for supersymmetric particles with R parity violating decays in $e^{+} e^{-}$collisions at $\sqrt{s}$ up to $209-\mathrm{GeV}, "$ Eur. Phys. J. C 31 (2003) 1-16, arXiv:hep-ex/0210014 [hep-ex].

[103] CDF Collaboration, "Search for Pair Production of Strongly Interacting Particles Decaying to Pairs of Jets in $p \bar{p}$ Collisions at $\sqrt{s}=1.96 \mathrm{TeV}, "$ Phys. Rev. Lett. 111 no. 3, (2013) 031802, arXiv:1303.2699 [hep-ex].

[104] Y. Bai, A. Katz, and B. Tweedie, "Pulling Out All the Stops: Searching for RPV SUSY with Stop-Jets," JHEP 01 (2014) 040, arXiv:1309.6631 [hep-ph].

[105] ATLAS Collaboration, "A search for R-parity violating scalar top decays in all-hadronic final states with the ATLAS detector in $\sqrt{s}=8 \mathrm{TeV} p p$ collisions," 2015. ATLAS-CONF-2015-026.

[106] S. Sapeta, "QCD and Jets at Hadron Colliders," Prog. Part. Nucl. Phys. 89 (2016) 1-55, arXiv:1511.09336 [hep-ph].

[107] ATLAS Collaboration, "Search for the Standard Model Higgs boson decaying into $b \bar{b}$ produced in association with top quarks decaying hadronically in $p p$ collisions at $\sqrt{s}=8$ TeV with the ATLAS detector," arXiv:1604.03812 [hep-ex].

[108] ATLAS Collaboration, "Expected performance of the ATLAS b-tagging algorithms in Run-2," Jul, 2015. https://cds.cern.ch/record/2037697. ATL-PHYS-PUB-2015-022.

[109] CMS Collaboration, "Identification of b-quark jets with the CMS experiment," J. Instrum. 8 no. arXiv:1211.4462. CMS-BTV-12-001. CERN-PH-EP-2012-262, (Nov, 2012) P04013. 67 p. https://cds.cern.ch/record/1494669. Comments: Submitted to the Journal of Instrumentation.

[110] ATLAS Collaboration, ATLAS, "Identification of high transverse momentum top quarks in $p p$ collisions at $\sqrt{s}=8 \mathrm{TeV}$ with the ATLAS detector,".

[111] K. Hamaguchi, S. P. Liew, and M. Stoll, "How to decontaminate overlapping fat jets," Phys. Rev. D 92 no. 1, (2015) 015012, arXiv:1505.02930 [hep-ph]. 
[112] ATLAS Collaboration, "Search for strong gravity in multijet final states produced in pp collisions at $\sqrt{s}=13 \mathrm{TeV}$ using the ATLAS detector at the LHC," JHEP 03 (2016) 026, arXiv:1512.02586 [hep-ex].

[113] P. F. M. Czakon and A. Mitov, "The total top quark pair production cross-section at hadron colliders through $\mathcal{O}\left(\alpha_{s}^{4}\right)$," Phys. Rev. Lett. 110 (2013) 252004, arXiv:1303.6254 [hep-ph].

[114] G. Bevilacqua, M. Czakon, C. G. Papadopoulos, R. Pittau, and M. Worek, "Assault on the NLO Wishlist: $p p \rightarrow$ t anti-t b anti-b," JHEP 09 (2009) 109, arXiv:0907.4723 [hep-ph].

[115] F. Maltoni, D. Pagani, and I. Tsinikos, "Associated production of a top-quark pair with vector bosons at NLO in QCD: impact on $t \bar{t} H$ searches at the LHC," arXiv: 1507.05640 [hep-ph].

[116] J. M. Campbell and R. K. Ellis, " $t \bar{t} W^{+-}$production and decay at NLO," JHEP 07 (2012) 052, arXiv:1204.5678 [hep-ph].

[117] A. Kulesza, L. Motyka, T. Stebel, and V. Theeuwes, "Soft gluon resummation for associated $t \bar{t} H$ production at the LHC," arXiv:1509.02780 [hep-ph].

[118] A. Buckley, J. Ferrando, S. Lloyd, K. Nordstrm, B. Page, M. Rfenacht, M. Schnherr, and G. Watt, "LHAPDF6: parton density access in the LHC precision era," Eur. Phys. J. C 75 (2015) 132, arXiv:1412.7420 [hep-ph]. 


\begin{tabular}{|c|c|c|c|c|c|c|c|}
\hline$\lambda_{33 i}^{\prime \prime}$ & $\mu[\mathrm{GeV}]$ & $m_{\tilde{t}}-m_{\chi}+[\mathrm{GeV}]$ & $\sigma(2 b 2 j)[\mathrm{pb}]$ & $\sigma(4 b 2 j)[\mathrm{pb}]$ & $\sigma(6 b 2 j)[\mathrm{pb}]$ & $\sigma(1 t 5 b 2 j)[\mathrm{pb}]$ & $\sigma(2 t 4 b 2 j)[\mathrm{pb}]$ \\
\hline \multirow{6}{*}{$10^{-1}$} & 400 & 201 & $4.38 \cdot 10^{-4}$ & $8.80 \cdot 10^{-3}$ & $4.19 \cdot 10^{-2}$ & $2.42 \cdot 10^{-5}$ & $2.70 \cdot 10^{-9}$ \\
\hline & 450 & 153 & $1.67 \cdot 10^{-3}$ & $2.16 \cdot 10^{-2}$ & $6.66 \cdot 10^{-2}$ & $1.05 \cdot 10^{-5}$ & $3.21 \cdot 10^{-10}$ \\
\hline & 500 & 106 & $4.17 \cdot 10^{-3}$ & $2.83 \cdot 10^{-2}$ & $4.63 \cdot 10^{-2}$ & $1.56 \cdot 10^{-6}$ & $1.47 \cdot 10^{-11}$ \\
\hline & 550 & 59 & $1.26 \cdot 10^{-2}$ & $2.87 \cdot 10^{-2}$ & $1.60 \cdot 10^{-2}$ & $4.79 \cdot 10^{-7}$ & $8.45 \cdot 10^{-12}$ \\
\hline & 600 & 11 & $3.01 \cdot 10^{-2}$ & $2.98 \cdot 10^{-3}$ & $7.29 \cdot 10^{-5}$ & $2.30 \cdot 10^{-8}$ & $3.23 \cdot 10^{-12}$ \\
\hline & 650 & -36 & $3.08 \cdot 10^{-2}$ & $7.02 \cdot 10^{-5}$ & $5.19 \cdot 10^{-8}$ & $6.36 \cdot 10^{-10}$ & $2.65 \cdot 10^{-12}$ \\
\hline \multirow{6}{*}{$10^{-2}$} & 400 & 194 & $6.92 \cdot 10^{-8}$ & $1.28 \cdot 10^{-4}$ & $5.72 \cdot 10^{-2}$ & $6.99 \cdot 10^{-5}$ & $1.67 \cdot 10^{-8}$ \\
\hline & 450 & 146 & $3.30 \cdot 10^{-7}$ & $3.88 \cdot 10^{-4}$ & $1.09 \cdot 10^{-1}$ & $4.93 \cdot 10^{-5}$ & $4.35 \cdot 10^{-9}$ \\
\hline & 500 & 100 & $1.30 \cdot 10^{-6}$ & $7.68 \cdot 10^{-4}$ & $1.09 \cdot 10^{-1}$ & $1.97 \cdot 10^{-5}$ & $6.37 \cdot 10^{-10}$ \\
\hline & 550 & 52 & $1.44 \cdot 10^{-5}$ & $2.57 \cdot 10^{-3}$ & $1.12 \cdot 10^{-1}$ & $7.86 \cdot 10^{-6}$ & $1.14 \cdot 10^{-10}$ \\
\hline & 600 & 5 & $2.40 \cdot 10^{-2}$ & $1.71 \cdot 10^{-2}$ & $2.96 \cdot 10^{-3}$ & $1.82 \cdot 10^{-7}$ & $4.10 \cdot 10^{-12}$ \\
\hline & 650 & -43 & $3.29 \cdot 10^{-2}$ & $6.68 \cdot 10^{-5}$ & $4.51 \cdot 10^{-8}$ & $5.88 \cdot 10^{-10}$ & $2.62 \cdot 10^{-12}$ \\
\hline \multirow{6}{*}{$10^{-3}$} & 400 & 194 & $6.96 \cdot 10^{-12}$ & $1.23 \cdot 10^{-6}$ & $5.40 \cdot 10^{-2}$ & $3.90 \cdot 10^{-3}$ & $7.06 \cdot 10^{-5}$ \\
\hline & 450 & 146 & $3.33 \cdot 10^{-11}$ & $3.77 \cdot 10^{-6}$ & $1.06 \cdot 10^{-1}$ & $3.53 \cdot 10^{-3}$ & $2.93 \cdot 10^{-5}$ \\
\hline & 500 & 99 & $1.32 \cdot 10^{-10}$ & $7.56 \cdot 10^{-6}$ & $1.09 \cdot 10^{-1}$ & $1.64 \cdot 10^{-3}$ & $6.28 \cdot 10^{-6}$ \\
\hline & 550 & 52 & $1.50 \cdot 10^{-9}$ & $2.58 \cdot 10^{-5}$ & $1.09 \cdot 10^{-1}$ & $6.71 \cdot 10^{-4}$ & $1.03 \cdot 10^{-6}$ \\
\hline & 600 & 5 & $1.08 \cdot 10^{-4}$ & $6.81 \cdot 10^{-3}$ & $9.82 \cdot 10^{-2}$ & $1.55 \cdot 10^{-4}$ & $6.16 \cdot 10^{-8}$ \\
\hline & 650 & -43 & $3.29 \cdot 10^{-2}$ & $6.51 \cdot 10^{-5}$ & $4.35 \cdot 10^{-8}$ & $5.85 \cdot 10^{-10}$ & $2.62 \cdot 10^{-12}$ \\
\hline \multirow{6}{*}{$10^{-4}$} & 400 & 194 & $6.96 \cdot 10^{-16}$ & $3.27 \cdot 10^{-9}$ & $3.85 \cdot 10^{-3}$ & $2.76 \cdot 10^{-2}$ & $4.96 \cdot 10^{-2}$ \\
\hline & 450 & 146 & $3.33 \cdot 10^{-15}$ & $1.62 \cdot 10^{-8}$ & $1.97 \cdot 10^{-2}$ & $6.57 \cdot 10^{-2}$ & $5.46 \cdot 10^{-2}$ \\
\hline & 500 & 99 & $1.32 \cdot 10^{-14}$ & $4.68 \cdot 10^{-8}$ & $4.13 \cdot 10^{-2}$ & $6.30 \cdot 10^{-2}$ & $2.40 \cdot 10^{-2}$ \\
\hline & 550 & 52 & $1.51 \cdot 10^{-13}$ & $2.05 \cdot 10^{-7}$ & $6.98 \cdot 10^{-2}$ & $4.28 \cdot 10^{-2}$ & $6.55 \cdot 10^{-3}$ \\
\hline & 600 & 5 & $1.22 \cdot 10^{-8}$ & $6.87 \cdot 10^{-5}$ & $9.68 \cdot 10^{-2}$ & $1.50 \cdot 10^{-2}$ & $6.22 \cdot 10^{-4}$ \\
\hline & 650 & -43 & $3.29 \cdot 10^{-2}$ & $6.30 \cdot 10^{-5}$ & $4.14 \cdot 10^{-8}$ & $5.75 \cdot 10^{-10}$ & $2.62 \cdot 10^{-12}$ \\
\hline \multirow{6}{*}{$10^{-5}$} & 400 & 194 & $6.96 \cdot 10^{-20}$ & $4.40 \cdot 10^{-13}$ & $6.97 \cdot 10^{-7}$ & $5.01 \cdot 10^{-4}$ & $8.98 \cdot 10^{-2}$ \\
\hline & 450 & 146 & $3.33 \cdot 10^{-19}$ & $2.81 \cdot 10^{-12}$ & $5.93 \cdot 10^{-6}$ & $1.97 \cdot 10^{-3}$ & $1.64 \cdot 10^{-1}$ \\
\hline & 500 & 99 & $1.32 \cdot 10^{-18}$ & $1.20 \cdot 10^{-11}$ & $2.71 \cdot 10^{-5}$ & $4.13 \cdot 10^{-3}$ & $1.57 \cdot 10^{-1}$ \\
\hline & 550 & 52 & $1.51 \cdot 10^{-17}$ & $9.73 \cdot 10^{-11}$ & $1.57 \cdot 10^{-4}$ & $9.63 \cdot 10^{-3}$ & $1.48 \cdot 10^{-1}$ \\
\hline & 600 & 5 & $1.22 \cdot 10^{-12}$ & $9.65 \cdot 10^{-8}$ & $1.91 \cdot 10^{-3}$ & $2.98 \cdot 10^{-2}$ & $1.16 \cdot 10^{-1}$ \\
\hline & 650 & -43 & $3.29 \cdot 10^{-2}$ & $6.26 \cdot 10^{-5}$ & $3.97 \cdot 10^{-8}$ & $5.54 \cdot 10^{-10}$ & $2.62 \cdot 10^{-12}$ \\
\hline \multirow{6}{*}{$10^{-6}$} & 400 & 194 & $6.96 \cdot 10^{-24}$ & $4.42 \cdot 10^{-17}$ & $7.03 \cdot 10^{-11}$ & $5.04 \cdot 10^{-6}$ & $9.04 \cdot 10^{-2}$ \\
\hline & 450 & 146 & $3.33 \cdot 10^{-23}$ & $2.83 \cdot 10^{-16}$ & $6.01 \cdot 10^{-10}$ & $2.00 \cdot 10^{-5}$ & $1.66 \cdot 10^{-1}$ \\
\hline & 500 & 99 & $1.32 \cdot 10^{-22}$ & $1.22 \cdot 10^{-15}$ & $2.80 \cdot 10^{-9}$ & $4.26 \cdot 10^{-5}$ & $1.63 \cdot 10^{-1}$ \\
\hline & 550 & 52 & $1.51 \cdot 10^{-21}$ & $1.01 \cdot 10^{-14}$ & $1.70 \cdot 10^{-8}$ & $1.04 \cdot 10^{-4}$ & $1.59 \cdot 10^{-1}$ \\
\hline & 600 & 5 & $1.22 \cdot 10^{-16}$ & $1.11 \cdot 10^{-11}$ & $2.53 \cdot 10^{-7}$ & $3.96 \cdot 10^{-4}$ & $1.54 \cdot 10^{-1}$ \\
\hline & 650 & -43 & $3.29 \cdot 10^{-2}$ & $6.26 \cdot 10^{-5}$ & $3.97 \cdot 10^{-8}$ & $5.53 \cdot 10^{-10}$ & $2.62 \cdot 10^{-12}$ \\
\hline \multirow{6}{*}{$10^{-7}$} & 400 & 194 & $6.96 \cdot 10^{-28}$ & $4.43 \cdot 10^{-21}$ & $7.01 \cdot 10^{-15}$ & $5.04 \cdot 10^{-8}$ & $9.05 \cdot 10^{-2}$ \\
\hline & 450 & 146 & $3.33 \cdot 10^{-27}$ & $2.83 \cdot 10^{-20}$ & $6.01 \cdot 10^{-14}$ & $2.00 \cdot 10^{-7}$ & $1.66 \cdot 10^{-1}$ \\
\hline & 500 & 99 & $1.32 \cdot 10^{-26}$ & $1.21 \cdot 10^{-19}$ & $2.80 \cdot 10^{-13}$ & $4.26 \cdot 10^{-7}$ & $1.63 \cdot 10^{-1}$ \\
\hline & 550 & 52 & $1.51 \cdot 10^{-25}$ & $1.01 \cdot 10^{-18}$ & $1.70 \cdot 10^{-12}$ & $1.04 \cdot 10^{-6}$ & $1.59 \cdot 10^{-1}$ \\
\hline & 600 & 5 & $1.22 \cdot 10^{-20}$ & $1.11 \cdot 10^{-15}$ & $2.54 \cdot 10^{-11}$ & $3.96 \cdot 10^{-6}$ & $1.54 \cdot 10^{-1}$ \\
\hline & 650 & -43 & $3.37 \cdot 10^{-2}$ & $6.31 \cdot 10^{-5}$ & $3.99 \cdot 10^{-8}$ & $5.54 \cdot 10^{-10}$ & $2.61 \cdot 10^{-12}$ \\
\hline
\end{tabular}

TABLE IV: Benchmark 1: production cross-section for $\sigma(p p \rightarrow \tilde{\tilde{t}} \rightarrow X)$ at $\sqrt{s}=14 \mathrm{TeV}$, where $X=2 b 2 j, 4 b 2 j, 6 b 2 j, 1 t 5 b 2 j$ and $2 t 4 b 2 j$, as a function of $\lambda_{33 i}^{\prime \prime}$ and for different values of $m_{\tilde{t}}-m_{\chi^{+}}$. See Tabs. II and III for the low-energy values of the MSSM parameters. 


\begin{tabular}{|c|c|c|c|c|c|c|c|}
\hline$\lambda_{33 i}^{\prime \prime}$ & $\mu[\mathrm{GeV}]$ & $m_{\tilde{t}}-m_{\chi}+[\mathrm{GeV}]$ & $\sigma(2 b 2 j)[\mathrm{pb}]$ & $\sigma(4 b 2 j)[\mathrm{pb}]$ & $\sigma(6 b 2 j)[\mathrm{pb}]$ & $\sigma(1 t 5 b 2 j)[\mathrm{pb}]$ & $\sigma(2 t 4 b 2 j)[\mathrm{pb}]$ \\
\hline \multirow{6}{*}{$10^{-1}$} & 750 & 243 & $3.52 \cdot 10^{-5}$ & $4.57 \cdot 10^{-4}$ & $1.45 \cdot 10^{-3}$ & $1.62 \cdot 10^{-6}$ & $4.88 \cdot 10^{-10}$ \\
\hline & 800 & 195 & $8.53 \cdot 10^{-5}$ & $7.56 \cdot 10^{-4}$ & $1.64 \cdot 10^{-3}$ & $8.09 \cdot 10^{-7}$ & $1.00 \cdot 10^{-10}$ \\
\hline & 850 & 147 & $2.63 \cdot 10^{-4}$ & $1.42 \cdot 10^{-3}$ & $1.87 \cdot 10^{-3}$ & $2.63 \cdot 10^{-7}$ & $9.83 \cdot 10^{-12}$ \\
\hline & 900 & 100 & $5.30 \cdot 10^{-4}$ & $1.38 \cdot 10^{-3}$ & $8.93 \cdot 10^{-4}$ & $5.12 \cdot 10^{-8}$ & $1.09 \cdot 10^{-12}$ \\
\hline & 950 & 52 & $1.02 \cdot 10^{-3}$ & $7.80 \cdot 10^{-4}$ & $1.48 \cdot 10^{-4}$ & $1.42 \cdot 10^{-8}$ & $5.78 \cdot 10^{-13}$ \\
\hline & 1000 & 5 & $1.43 \cdot 10^{-3}$ & $2.02 \cdot 10^{-5}$ & $7.30 \cdot 10^{-8}$ & $2.66 \cdot 10^{-10}$ & $3.51 \cdot 10^{-13}$ \\
\hline \multirow{6}{*}{$10^{-2}$} & 750 & 239 & $5.34 \cdot 10^{-9}$ & $6.72 \cdot 10^{-6}$ & $1.89 \cdot 10^{-3}$ & $2.53 \cdot 10^{-6}$ & $7.96 \cdot 10^{-10}$ \\
\hline & 800 & 191 & $1.70 \cdot 10^{-8}$ & $1.45 \cdot 10^{-5}$ & $2.79 \cdot 10^{-3}$ & $1.57 \cdot 10^{-6}$ & $2.19 \cdot 10^{-10}$ \\
\hline & 850 & 143 & $8.74 \cdot 10^{-8}$ & $4.39 \cdot 10^{-5}$ & $5.35 \cdot 10^{-3}$ & $9.25 \cdot 10^{-7}$ & $4.05 \cdot 10^{-11}$ \\
\hline & 900 & 96 & $3.85 \cdot 10^{-7}$ & $9.09 \cdot 10^{-5}$ & $5.08 \cdot 10^{-3}$ & $3.46 \cdot 10^{-7}$ & $6.14 \cdot 10^{-12}$ \\
\hline & 950 & 48 & $4.94 \cdot 10^{-6}$ & $3.13 \cdot 10^{-4}$ & $4.64 \cdot 10^{-3}$ & $1.95 \cdot 10^{-7}$ & $2.18 \cdot 10^{-12}$ \\
\hline & 1000 & 1 & $1.47 \cdot 10^{-3}$ & $1.22 \cdot 10^{-5}$ & $2.63 \cdot 10^{-8}$ & $1.67 \cdot 10^{-10}$ & $3.47 \cdot 10^{-13}$ \\
\hline \multirow{6}{*}{$10^{-3}$} & 750 & 239 & $5.37 \cdot 10^{-13}$ & $6.38 \cdot 10^{-8}$ & $1.90 \cdot 10^{-3}$ & $3.35 \cdot 10^{-5}$ & $1.50 \cdot 10^{-7}$ \\
\hline & 800 & 191 & $1.71 \cdot 10^{-12}$ & $1.38 \cdot 10^{-7}$ & $2.77 \cdot 10^{-3}$ & $3.15 \cdot 10^{-5}$ & $8.94 \cdot 10^{-8}$ \\
\hline & 850 & 143 & $8.88 \cdot 10^{-12}$ & $4.26 \cdot 10^{-7}$ & $5.11 \cdot 10^{-3}$ & $3.65 \cdot 10^{-5}$ & $6.51 \cdot 10^{-8}$ \\
\hline & 900 & 96 & $3.98 \cdot 10^{-11}$ & $9.04 \cdot 10^{-7}$ & $5.16 \cdot 10^{-3}$ & $2.27 \cdot 10^{-5}$ & $2.49 \cdot 10^{-8}$ \\
\hline & 950 & 48 & $5.57 \cdot 10^{-10}$ & $3.41 \cdot 10^{-6}$ & $5.17 \cdot 10^{-3}$ & $1.25 \cdot 10^{-5}$ & $7.57 \cdot 10^{-9}$ \\
\hline & 1000 & 1 & $1.47 \cdot 10^{-3}$ & $1.18 \cdot 10^{-5}$ & $2.57 \cdot 10^{-8}$ & $1.90 \cdot 10^{-10}$ & $4.29 \cdot 10^{-13}$ \\
\hline \multirow{6}{*}{$10^{-4}$} & 750 & 239 & $5.37 \cdot 10^{-17}$ & $3.70 \cdot 10^{-10}$ & $6.38 \cdot 10^{-4}$ & $1.07 \cdot 10^{-3}$ & $4.49 \cdot 10^{-4}$ \\
\hline & 800 & 191 & $1.71 \cdot 10^{-16}$ & $9.37 \cdot 10^{-10}$ & $1.28 \cdot 10^{-3}$ & $1.40 \cdot 10^{-3}$ & $3.82 \cdot 10^{-4}$ \\
\hline & 850 & 143 & $8.89 \cdot 10^{-16}$ & $3.26 \cdot 10^{-9}$ & $2.99 \cdot 10^{-3}$ & $2.11 \cdot 10^{-3}$ & $3.71 \cdot 10^{-4}$ \\
\hline & 900 & 96 & $3.98 \cdot 10^{-15}$ & $7.58 \cdot 10^{-9}$ & $3.61 \cdot 10^{-3}$ & $1.58 \cdot 10^{-3}$ & $1.73 \cdot 10^{-4}$ \\
\hline & 950 & 48 & $5.58 \cdot 10^{-14}$ & $3.06 \cdot 10^{-8}$ & $4.21 \cdot 10^{-3}$ & $1.02 \cdot 10^{-3}$ & $6.12 \cdot 10^{-5}$ \\
\hline & 1000 & 1 & $1.11 \cdot 10^{-3}$ & $9.79 \cdot 10^{-6}$ & $2.36 \cdot 10^{-8}$ & $2.38 \cdot 10^{-9}$ & $6.02 \cdot 10^{-11}$ \\
\hline \multirow{6}{*}{$10^{-5}$} & 750 & 239 & $5.37 \cdot 10^{-21}$ & $8.67 \cdot 10^{-14}$ & $3.50 \cdot 10^{-7}$ & $5.88 \cdot 10^{-5}$ & $2.47 \cdot 10^{-3}$ \\
\hline & 800 & 191 & $1.71 \cdot 10^{-20}$ & $2.84 \cdot 10^{-13}$ & $1.18 \cdot 10^{-6}$ & $1.29 \cdot 10^{-4}$ & $3.51 \cdot 10^{-3}$ \\
\hline & 850 & 143 & $8.89 \cdot 10^{-20}$ & $1.34 \cdot 10^{-12}$ & $5.02 \cdot 10^{-6}$ & $3.54 \cdot 10^{-4}$ & $6.22 \cdot 10^{-3}$ \\
\hline & 900 & 96 & $3.98 \cdot 10^{-19}$ & $4.44 \cdot 10^{-12}$ & $1.24 \cdot 10^{-5}$ & $5.41 \cdot 10^{-4}$ & $5.92 \cdot 10^{-3}$ \\
\hline & 950 & 48 & $5.58 \cdot 10^{-18}$ & $2.89 \cdot 10^{-11}$ & $3.75 \cdot 10^{-5}$ & $9.01 \cdot 10^{-4}$ & $5.43 \cdot 10^{-3}$ \\
\hline & 1000 & 1 & $5.75 \cdot 10^{-6}$ & $1.40 \cdot 10^{-7}$ & $9.36 \cdot 10^{-10}$ & $8.84 \cdot 10^{-9}$ & $2.09 \cdot 10^{-8}$ \\
\hline \multirow{6}{*}{$10^{-6}$} & 750 & 239 & $5.37 \cdot 10^{-25}$ & $8.79 \cdot 10^{-18}$ & $3.60 \cdot 10^{-11}$ & $6.04 \cdot 10^{-7}$ & $2.54 \cdot 10^{-3}$ \\
\hline & 800 & 191 & $1.71 \cdot 10^{-24}$ & $2.90 \cdot 10^{-17}$ & $1.23 \cdot 10^{-10}$ & $1.34 \cdot 10^{-6}$ & $3.67 \cdot 10^{-3}$ \\
\hline & 850 & 143 & $8.89 \cdot 10^{-24}$ & $1.38 \cdot 10^{-16}$ & $5.34 \cdot 10^{-10}$ & $3.77 \cdot 10^{-6}$ & $6.62 \cdot 10^{-3}$ \\
\hline & 900 & 96 & $3.98 \cdot 10^{-23}$ & $4.66 \cdot 10^{-16}$ & $1.36 \cdot 10^{-9}$ & $5.98 \cdot 10^{-6}$ & $6.55 \cdot 10^{-3}$ \\
\hline & 950 & 48 & $5.58 \cdot 10^{-22}$ & $3.15 \cdot 10^{-15}$ & $4.46 \cdot 10^{-9}$ & $1.07 \cdot 10^{-5}$ & $6.47 \cdot 10^{-3}$ \\
\hline & 1000 & 1 & $6.53 \cdot 10^{-10}$ & $1.84 \cdot 10^{-11}$ & $1.42 \cdot 10^{-13}$ & $1.34 \cdot 10^{-10}$ & $3.17 \cdot 10^{-8}$ \\
\hline \multirow{6}{*}{$10^{-7}$} & 750 & 239 & $5.37 \cdot 10^{-29}$ & $8.80 \cdot 10^{-22}$ & $3.60 \cdot 10^{-15}$ & $6.04 \cdot 10^{-9}$ & $2.54 \cdot 10^{-3}$ \\
\hline & 800 & 191 & $1.71 \cdot 10^{-28}$ & $2.90 \cdot 10^{-21}$ & $1.23 \cdot 10^{-14}$ & $1.34 \cdot 10^{-8}$ & $3.67 \cdot 10^{-3}$ \\
\hline & 850 & 143 & $8.89 \cdot 10^{-28}$ & $1.38 \cdot 10^{-20}$ & $5.35 \cdot 10^{-14}$ & $3.78 \cdot 10^{-8}$ & $6.59 \cdot 10^{-3}$ \\
\hline & 900 & 96 & $3.98 \cdot 10^{-27}$ & $4.66 \cdot 10^{-20}$ & $1.37 \cdot 10^{-13}$ & $5.98 \cdot 10^{-8}$ & $6.54 \cdot 10^{-3}$ \\
\hline & 950 & 48 & $5.58 \cdot 10^{-26}$ & $3.16 \cdot 10^{-19}$ & $4.47 \cdot 10^{-13}$ & $1.08 \cdot 10^{-7}$ & $6.49 \cdot 10^{-3}$ \\
\hline & 1000 & 1 & $6.54 \cdot 10^{-14}$ & $1.85 \cdot 10^{-15}$ & $1.43 \cdot 10^{-17}$ & $1.35 \cdot 10^{-12}$ & $3.19 \cdot 10^{-8}$ \\
\hline
\end{tabular}

TABLE V: Benchmark 2: production cross-section for $\sigma(p p \rightarrow \tilde{\tilde{t}} \rightarrow X)$ at $\sqrt{s}=14 \mathrm{TeV}$, where $X=2 b 2 j, 4 b 2 j, 6 b 2 j, 1 t 5 b 2 j$ and $2 t 4 b 2 j$, as a function of $\lambda_{33 i}^{\prime \prime}$ and for different values of $m_{\tilde{t}}-m_{\chi^{+}}$. See Tabs. II and III for the low-energy values of the MSSM parameters. 